\title{
Petrogenesis of alkaline basalt-hosted sapphire megacrysts. Petrological and geochemical investigations of in situ sapphire occurrences from the Siebengebirge Volcanic Field, Germany
}

\author{
L. C. Baldwin ${ }^{1,2} \cdot$ F. Tomaschek ${ }^{1} \cdot$ C. Ballhaus ${ }^{1} \cdot$ A. Gerdes $^{3} \cdot$ R. O. C. Fonseca ${ }^{1}$

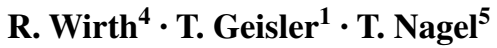

Received: 13 January 2017 / Accepted: 18 April 2017 / Published online: 23 May 2017

(C) The Author(s) 2017. This article is an open access publication

\begin{abstract}
Megacrystic sapphires are frequently associated with alkaline basalts, most notably in Asia and Australia, although basalt is not generally normative in corundum. Most of these sapphire occurrences are located in alluvial or eluvial deposits, making it difficult to study the enigmatic relationship between the sapphires and their host rocks. Here, we present detailed petrological and geochemical investigations of in situ megacrystic sapphires within alkaline basalts from the Cenozoic Siebengebirge Volcanic Field (SVF) in Germany. Markedly, the sapphires show several micrometer thick spinel coronas at the contact with the host basalt, indicating chemical disequilibrium between the sapphire and the basaltic melt, supporting a xenogenetic relationship. However, in situ U-Pb dating of a Columbite Group inclusion within one Siebengebirge sapphire using laser ablation-inductively coupled plasma-mass spectrometry (LA-ICP-MS) indicates a close genetic relationship between sapphire crystallization and alkaline mafic volcanism in the SVF. The syngenetic mineral inclusion suite
\end{abstract}

Communicated by Timothy L. Grove.

L. C. Baldwin

lisa.baldwin@univie.ac.at

1 Steinmann Institute, University of Bonn, Meckenheimer Allee 169, 53111 Bonn, Germany

2 Department of Lithospheric Research, University of Vienna, Althanstraße 14, 1090 Vienna, Austria

3 Geozentrum, Goethe University Frankfurt, Altenhöferalle 1, 60438 Frankfurt, Germany

4 Helmholz Zentrum Potsdam, Telegrafenberg, 14473 Potsdam, Germany

5 Department of Geoscience, University of Aarhus, Høegh-Guldbergs Gade 2, 8000 Aarhus C, Denmark including carbonates, members of the Pyrochlore, Betafite and Columbite Groupe minerals, as well as a high abundance of HFSE and of gaseous low-density $\mathrm{CO}_{2}$ inclusions support a parentage of a highly evolved, $\mathrm{MgO}$ and $\mathrm{FeO}$ deficient carbonatitic melt. We identified $\mathrm{CO}_{2}$ to be the link between alkaline basaltic volcanism and the xenocrystic sapphires. Only alkaline volcanic suites can build up enough $\mathrm{CO}_{2}$ in this magma chamber upon fractionation so that at high degrees of fractionation a carbonatitic melt exsolves which in turn can crystallize sapphires.

Keywords Sapphire $\cdot$ Alkaline basalt $\cdot$ Siebengebirge Volcanic Field · Columbite Group mineral dating · Carbonatite

\section{Introduction}

Sapphire and ruby are gem varieties of corundum $\left(\alpha-\mathrm{Al}_{2} \mathrm{O}_{3}\right)$ and belong to the most prominent and precious gemstones, along with diamond and emerald. Deposits of gem-quality corundum are well distributed around the world (e.g., Hughes 1997). A review of the literature on gem corundum shows that corundum occurs in various geological settings and seems to be generated via several different petrogenetic processes, such as metamorphic reactions or through igneous crystallization from aluminarich magmas (see references in this work and references therein). As to the broad variability in the depositional type of corundum, Simonet et al. (2008) established a classification scheme according to their geological occurrence, distinguishing between primary and secondary corundum deposits. Primary deposits are those where corundum is entrained inside the same rock that it crystallized from. In secondary deposits, corundum is an inherited mineral that 
formed in a different petrogenetic setting as to where it is deposited.

Of special interest are secondary corundum deposits that are associated with rift-related alkaline basaltic volcanism as these are among the world's largest gem corundum suppliers (e.g., Guo et al. 1996; Limtrakun et al. 2001; Shore and Weldon 2009) and are hence economically of great importance. Such basaltic secondary deposits are located all over the world, most notably in Asia (e.g., Guo et al. 1996, Giuliani et al. 2015), and in eastern Australia, at the western Pacific margin (e.g., Coenraads 1990; Graham et al. 2008; Sutherland 1996; Sutherland et al. 1998a, 2002, 2009b, 2015, 2017; Saminpanya 2001; McGee 2005; Abduriyim et al. 2012), but also in Europe (e.g., Malikova 1999; Giuliani et al. 2009; Uher et al. 2012), Africa (Kiefert and Schmetzer 1987; Krzemnicki et al. 1996; Schwarz et al. 2000; Pardieux et al. 2014), in South America (Sutherland et al. 2008), and in North America (e.g., Garland 2002; Berg 2007, 2014; Palke et al. 2017). In most basalt fields, corundum is present as blue, green, or yellow sapphires, and is commonly referred to as blue-green-yellow (BGY) sapphire suite (e.g., Sutherland et al. 1998a, b). These BGY sapphires may reach up to $5 \mathrm{~cm}$ in diameter and usually occur in alluvial or eluvial placer deposits. In situ occurrences where the sapphires are still inside their host basalts are rarely found (e.g., MacNevin 1972; Stephenson 1976; Vichit 1987). The best known example is a basalt flow at Changle, China (Guo et al. 1992; Zelong et al. 2007; Giuliani et al. 2015), while numerous corundum, including gem crystals, were recorded in extensive flows south of Jos Plateau, Nigeria (Wright 1985).

Although this type of corundum deposit is economically the most valuable amongst corundum deposits, the origin of these corundum is to date still poorly understood (e.g., Izokh et al. 2010; Uher et al. 2012). The problem is that primitive alkali basalts are not corundum-normative when they leave their mantle sources, regardless of the pressure of formation (Liu and Presnall 1990). Hence, unless these sapphires precipitated via some yet unknown process out of the basaltic melts, they must instead have a xenogenetic relation with their host rocks. Indeed, anhedral crystal shapes, rounded edges, and macroscopically visible spinel coronas at the sapphire-basalt interface (e.g., Stephenson 1976; Guo et al. 1996; Sutherland et al. 2002; McGee 2005) indicate disequilibrium between these sapphires and their host rocks, supporting a xenocrystic nature. There seems to be a consensus (see references in this work) that the sapphires originate from a reservoir in the mantle or the crust, and were later transported to the surface by ascending basaltic melts. Yet, the origin of the basalt-associated sapphires remains a puzzle. Moreover, it is still an open question as to why sapphires most often occur as xenocrystals in alkaline basalts from intra-continental volcanic fields, but only rarely in $\mathrm{SiO}_{2}$ saturated rocks (e.g., Berger and Berg 2006; Berg 2007, 2014; Palke et al. 2017). The petrogenesis of alkaline basalt-hosted sapphires was intensively studied from various gem fields in the 1990s and early 2000s. These studies revealed that the sapphires that were brought to the surface by basaltic eruptions may have various pre-depositional histories and may be of metamorphic, magmatic, or of metasomatic origin (e.g., Sutherland et al. 2009a; Giuliani et al. 2014), depending on the $\delta^{18} \mathrm{O}$ values (e.g., Giuliani et al. 2005, 2007; Yui et al. 2006; Sutherland et al. 2009a, b), the mineral inclusion suite (e.g., Guo et al. 1996), their trace element geochemistry (Sutherland et al. 2009b, 2017), and most notably on the Ga/Mg ratio (Peucat et al. 2007).

Several hypotheses have been proposed to explain the igneous crystallization of sapphires, the most prominent being: (1) Crystallization from highly evolved melts such as syenites (e.g., Irving 1986; Coenraads et al. 1990; Aspen et al. 1990; Garnier et al. 2005; Giuliani et al. 2009). (2) Crystallization from alkaline $\mathrm{Si}$ - and $\mathrm{Al}$-rich melts formed by partial melting of amphibole-bearing lithospheric mantle (Sutherland 1996; Sutherland et al. 1998b). (3) Growth as restitic component during partial melting of Al-rich crustal protoliths such as anorthosites (Palke et al. 2016, 2017). (4) Hybrid formation where sapphire is stabilized by reaction of a fractionated silicate with a carbonatitic melt at mid-crustal levels (Guo et al. 1996). This hybrid model is based on the occurrence of a very complex mineral inclusion suite in sapphire that must have precipitated from melts of two different compositions, most probably a carbonatitic and a highly evolved silicate melt. Guo et al. (1996) proposed that evolved granite/syenitic pegmatite silicate melts become $\mathrm{Al}_{2} \mathrm{O}_{3}$ oversaturated in response to mixing with carbonatitic melts, resulting in local precipitation of corundum in the hybridization zone. (5) Crystallization at shallow crustal levels from an iron-rich syenitic melt with the participation of a carbonate- $\mathrm{H}_{2} \mathrm{O}-\mathrm{CO}_{2}$ fluid phase (Izokh et al. 2010). This model is similar to the model proposed by Guo et al. (1996) but does not include the participation of a carbonatitic melt. Izokh et al. (2010) rather suggested that the syenitic melt must have had initially high $\mathrm{CO}_{2}$ contents, as it was formed by differentiation of a $\mathrm{CO}_{2}$-rich basaltic melt. Differentiation of these $\mathrm{CO}_{2}$ rich syenitic magmas led to the formation of alkalicarbonate complexes, so that the residual melt becomes oversaturated in alumina and corundum may precipitate in the most fractionated magma chambers.

In this paper, we present a detailed microchemical investigation of up to $2 \mathrm{~cm}$ sized sapphire xenocrysts that occur within primitive alkali basalts and basanites from the Siebengebirge Volcanic Field (SVF) in Germany. The sapphires from the SVF are found in situ within alkali basalt, a relatively rare occurrence with the potential for providing 
important insights into how this type of sapphire formed. To date, there are only limited investigations of sapphire that are still attached to their host rocks using modern analytical techniques. Hence, the Siebengebirge sapphires provide an exceptional opportunity to study the genetical relationship of the sapphires and their host basalt. We present a unique petrogenetic model for the origin of the Siebengebirge sapphires that may provide important implications for the general origin of basalt-hosted sapphires and for predicting sites of possible further deposits.

\section{Geological setting}

The Siebengebirge Volcanic Field (SVF) is part of the Central European Volcanic Province (CEVP). The latter forms a 200-300 km broad Cenozoic intra-continental volcanic belt north of the Alpine Orogen. In Germany, volcanic fields belonging to the CEVP are (from west to east) the Eifel, the Siebengebirge, the Westerwald, the Vogelsberg, the Hessian Depression, the Rhön area, Heldburg, and the Oberpfalz (Fig. 1). Further European regions belonging to the CEVP are the Bohemian Massif in the Czech Republic, the Eger Rift System in the Czech Republic and Poland, the Pannonian Basin in Hungary, and the French Massif Central. Igneous activity in the CEVP lasted throughout the entire Cenozoic (Wilson and Downes 1991).

The SVF is located at the SE margin of the Lower Rhine Graben and is part of the Rhine rift system that formed in Eocene to Oligocene times as a consequence of syn- to post-orogenic extension in the Alpine foreland (Ziegler 1992). The NW-SE-trending Lower Rhine Graben cuts into the Rhenish Shield that is a Variscan tectonic block within the Rhenohercynian Zone and has been uplifted since Eocene times (e.g., Illies et al. 1979; Ziegler 1992; Oncken et al. 1999).

The total volcanic area of the SVF covers an area of about $900 \mathrm{~km}^{2}$. The volcanic activity in the Siebengebirge occurred over several eruptional phases, resulting in the eruption of a very broad spectrum of both $\mathrm{SiO}_{2}$ saturated
Fig. 1 Volcanic Fields of the Central European Volcanic Province (modified after Wedepohl et al. 1994). The main sampling localities Petersberg, Oelberg, and Jungfernberg are shown in the boxbox at the bottom of the figure

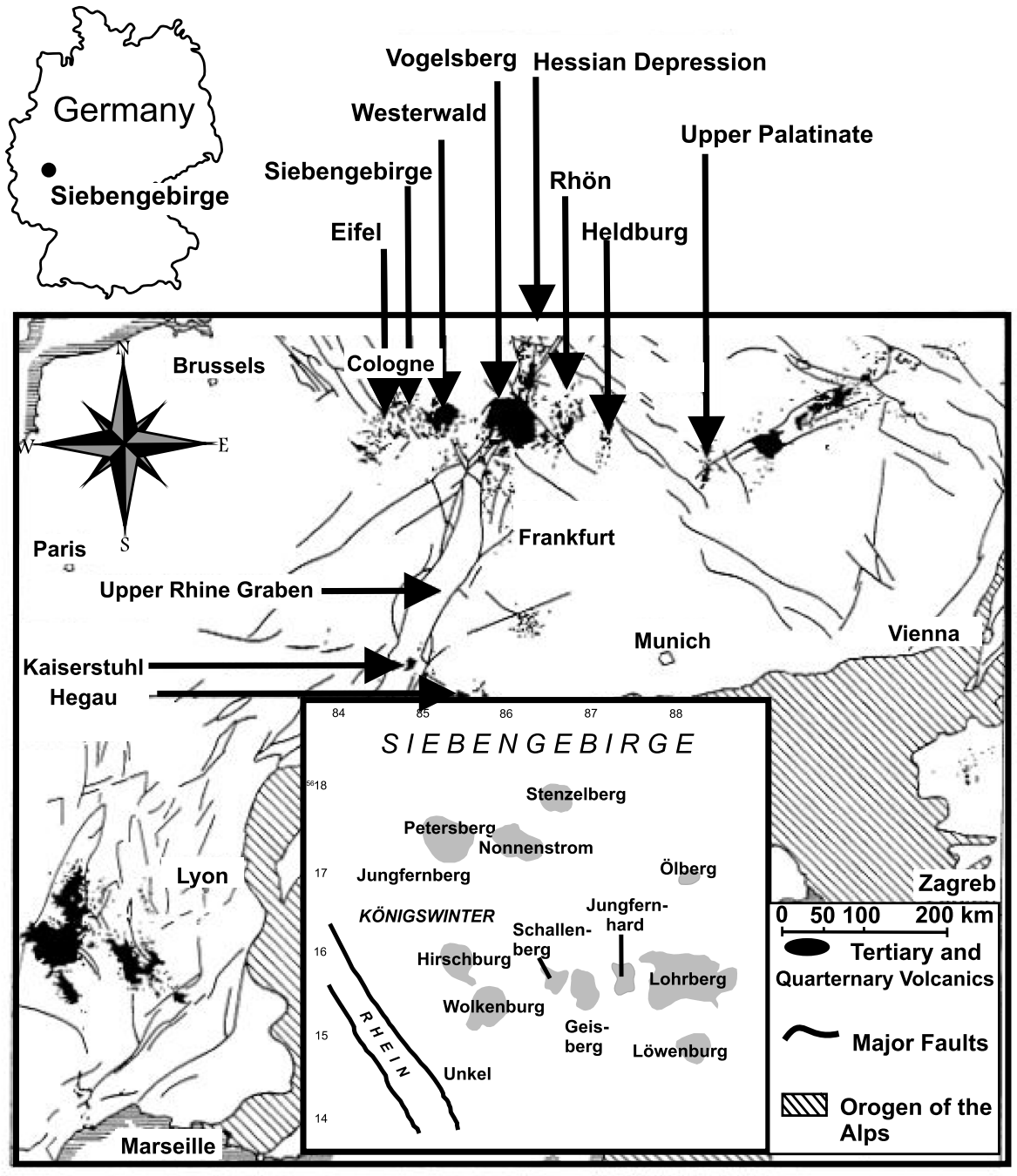


and $\mathrm{SiO}_{2}$ undersaturated mafic and felsic melts. The most primitive mafic volcanics are basanites to alkali basalts; more felsic volcanics are $\mathrm{SiO}_{2}$ saturated latites to trachytes and $\mathrm{SiO}_{2}$ undersaturated phonotephrites to tephriphonolites (Vieten et al. 1988; Kolb et al. 2012; Jung et al. 2012). The period of volcanic activity in the SVF has been dated using the K/Ar and Ar/Ar chronometers and lasted from 30 to $19 \mathrm{Ma}$ (Todt and Lippolt 1980; Linthout et al. 2009; Przybyla 2013). The main magmatic activity is thought to have occurred between 26 and 24 Ma (Przybyla 2013).

Alkaline basalts from the SVF have long been known to host megacrystic sapphires. They were described scientifically in the late nineteenth and early twentieth century (Brauns 1922, and references therein). However, until now, no effort has been made to investigate the sapphires entrained in the alkaline mafic rocks from the SVF with modern techniques and to identify possible genetic links to their host basalts.

\section{Samples}

Most of the sapphires studied in this work belong to the mineralogical collection of the Geological and Mineralogical Department of the University of Bonn and were recovered in the early twentieth century when the basalt occurrences (now protected) were still subject to quarrying. Other samples were donated by local collectors. The principal localities in the Siebengebirge from which sapphires were recovered are the Petersberg and Oelberg alkaline mafic rocks. Other known localities are the Jungfernberg in Heisterbacher Rott at the northern part of the Siebengebirge, and Unkel at the south-western extension of the Siebengebirge.

Samples studied included 11 sapphires with an anhedral to subhedral crystal shape and sizes of 5-20 mm. Sample descriptions, localities, and host rock lithologies are summarized in Table 1. Colors vary from a

Table 1 Petrographic description of the Siebengebirge sapphires, their host rock locality and locations within the Siebengebirge

\begin{tabular}{|c|c|c|c|c|c|c|c|}
\hline Sample & Host rock locality & $\begin{array}{l}\text { Host rock lithol- } \\
\text { ogy }\end{array}$ & Color & Size $(\mathrm{mm})$ & Shape & $\begin{array}{l}\text { Reaction rim } \\
\text { thickness }(\mu \mathrm{m})\end{array}$ & Inclusions \\
\hline 3 & Jungfernberg & Basanite & pale-blue, milky & 3 & Irregular & $70-80$ & $\begin{array}{l}\text { Calcite, Nb-rich } \\
\text { rutile, alkali } \\
\text { feldspar, spinel, } \\
\text { apatite, ilmenite }\end{array}$ \\
\hline 15 & Oelberg & Alkali Basalt & & 10 & Irregular & $40-100$ & Calcite \\
\hline 26 & Oelberg & Alkali Basalt & Dark blue & 10 & Irregular & $\begin{array}{l}50-100, \\
\text { Partly missing }\end{array}$ & $\begin{array}{l}\text { Calcite, plagioclase } \\
\text { series, alkali } \\
\text { feldspar, } \\
\text { Columbite Group, } \\
\text { thorite, thorianite, } \\
\text { apatite }\end{array}$ \\
\hline KS21 & Heisterbacher Rott & Alkali Basalt & $\begin{array}{l}\text { a) Pale blue, } \\
\text { milky; } \\
\text { b) Dark blue, clear }\end{array}$ & 7 & Irregular & $\begin{array}{l}10-50, \\
\text { Partly missing }\end{array}$ & - \\
\hline OL25 & Oelberg & Hawaiite & Pale blue, milky & 4 & Irregular & $100-200$ & $\begin{array}{l}\text { Calcite, } \mathrm{Nb} \text {-rich } \\
\text { rutile, ilmenite- } \\
\text { pyrophanite }\end{array}$ \\
\hline OL60 & Oelberg & Hawaiite & Pale blue, milky & 3 & Irregular & $\begin{array}{l}100-150, \text { one area } \\
\text { with } 500\end{array}$ & $\begin{array}{l}\text { Plagioclase series, } \\
\text { spinel, calcite }\end{array}$ \\
\hline OL61 & Oelberg & Hawaiite & Dark blue, clear & 3 & Irregular & ca. 100 & Plagioclase series \\
\hline UN31 & Unkel & Alkali Basalt & $\begin{array}{l}\text { Pale blue, milky } \\
\text { and dark blue } \\
\text { areas }\end{array}$ & 5 & Irregular & $40-100$ & - \\
\hline PE21 & Petersberg & Alkali Basalt & $\begin{array}{l}\text { Pale blue, milky } \\
\text { with gray area in } \\
\text { the center }\end{array}$ & 17 & Hypidiomorphic & $100-200$ & $\begin{array}{l}\mathrm{Nb} \text {-rich } \\
\text { microinclusions in } \\
\text { reaction rim }\end{array}$ \\
\hline PE25 & Petersberg & Alkali Basalt & $\begin{array}{l}\text { Pale blue, milky } \\
\text { with gray area in } \\
\text { one edge }\end{array}$ & 15 & Hypidiomorphic & $100-200$ & $\begin{array}{l}\text { Alkali feldspar, } \\
\text { Nb-rich } \\
\text { microinclusions in } \\
\text { reaction rim }\end{array}$ \\
\hline S52 & Unknown & - & Dark blue & 8 & Irregular & $10-300$ & $\begin{array}{l}\text { Plagioclase series, } \\
\text { alkali feldspar, } \\
\text { calcite }\end{array}$ \\
\hline
\end{tabular}


milky pale-blue to a clear dark blue, and two sapphires have large brown to gray domains (Fig. 2), distinguishing them macroscopically from the other samples. All sapphires were still attached to their host rocks and showed evidence for chemical disequilibrium with their host basalt, including resorbed edges, rounded shapes, and macroscopically visible dark green spinel coronas (Fig. 3a, b). The contact between sapphire and spinel is

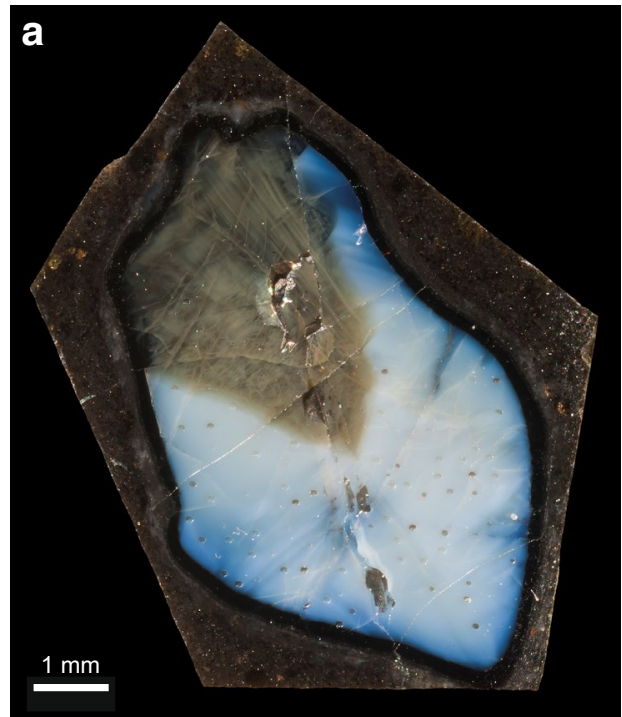

Fig. 2 Sapphire PE25 and graphical interpretation. a Photograph of sapphire PE25. b Graphical interpretation. The brownish area is chemically characterized by a higher enrichment in the HFSE $(\mathrm{Nb}$, $\mathrm{Ta}, \mathrm{Zr}$, and $\mathrm{Hf}$ ) compared to the blue area. The transition from the brown towards the blue area is extremely sharp. The black rim sur- quite sharp, but in their contact with the basaltic matrix the spinel coronas become more ragged and porous, and tend to disintegrate to discrete grains (Fig. 3a). The basalt in direct contact with the spinel coronas is enriched in plagioclase and depleted in modal pyroxene and titanomagnetite (Fig. 3b, c) compared to the average basaltic matrix further away from the sapphire. Most sapphires contain mineral and/or fluid inclusions. Except for two

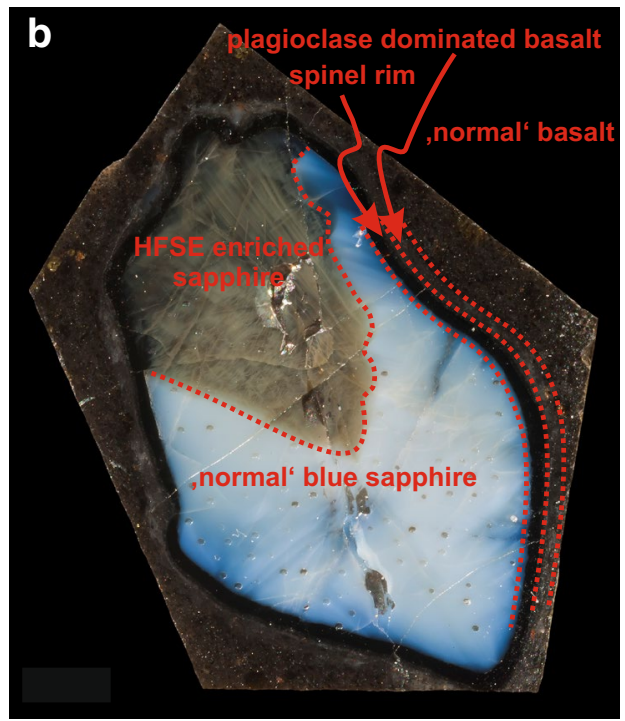

rounding the sapphire is spinel. The spinel rim is surrounded by a corona of plagioclase-rich basalt that has about the same thickness as the spinel rim. The plagioclase enriched basaltic matrix is surrounded by 'normal' basaltic matrix comprising plagioclase, pyroxene, and Tiphases
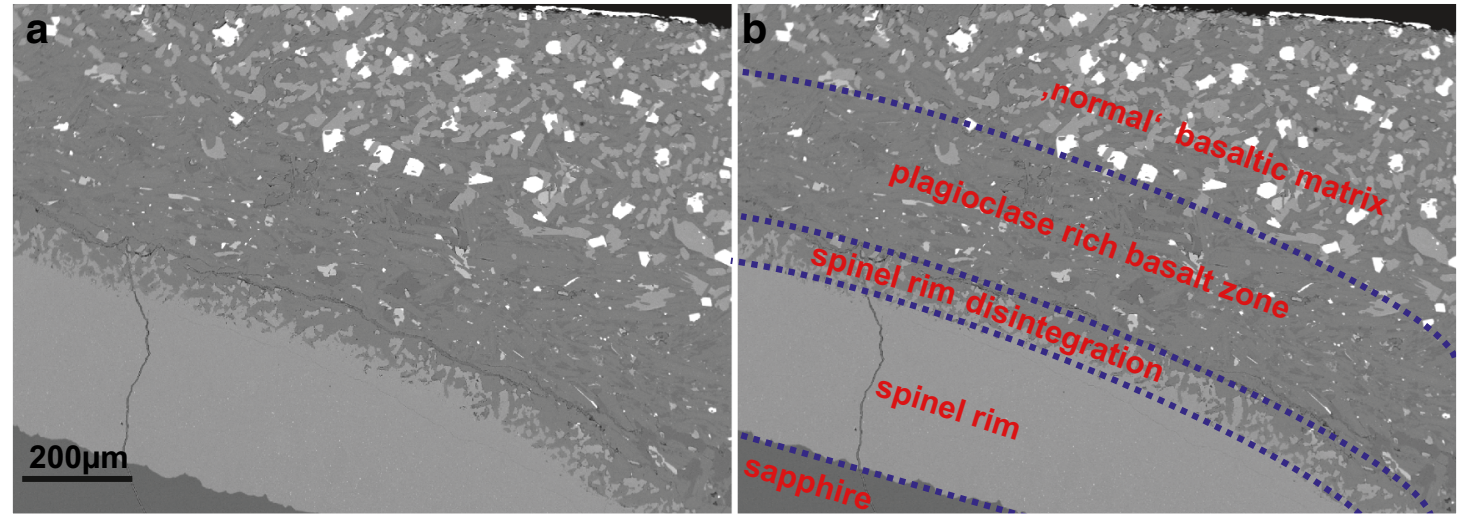

Fig. 3 a Backscattered electron image of the transition from the sapphire to the basaltic matrix. b Graphical interpretation of a, emphasizing the zonal structure of the spinel rim. The sapphire-spinel interface is well-defined whereas the transition zone from the spinel towards the basaltic melt occurs via a zone of disintegrated spinels that mingle in more or less equal fractions with the basaltic matrix. The basaltic matrix in direct contact with the spinel rim comprises almost pure plagioclase. $\mathrm{Fe}-\mathrm{Mg}$ phases such as pyroxene are missing. The plagioclase-rich basaltic matrix is followed by the 'normal' basaltic matrix with abundant plagioclase, pyroxene, and Ti-phases 
samples (Fig. 3d), the spinel coronas are virtually free of inclusions.

\section{Analytical methods}

\section{EPMA}

The sapphires, their reaction rims, and mineral inclusions were analyzed for major and minor elements with a JEOL JXA 8900 electron probe microanalyzer equipped with a tungsten cathode at the Steinmann Institute, University of Bonn. All measurements were performed at $15 \mathrm{kV}$ acceleration voltage and a probe current of $15 \mathrm{nA}$. The major elements were quantified on the $\mathrm{K} \alpha$ lines. Where possible, the trace elements $\mathrm{Nb}, \mathrm{Ce}, \mathrm{Y}, \mathrm{Ta}$, and $\mathrm{Zr}$ were quantified on the $\mathrm{L} \alpha$ lines, while $\mathrm{Th}, \mathrm{U}$, and $\mathrm{Pb}$ were quantified on their $\mathrm{M} \alpha$ lines. For the silicates, standards used for calibration are olivine for $\mathrm{Mg}, \mathrm{Fe}$, and $\mathrm{Si}$, jadeite for $\mathrm{Na}$, microcline for $\mathrm{K}$, anorthite for $\mathrm{Ca}$, augite for $\mathrm{Al}$, rutile for $\mathrm{Ti}, \mathrm{MnFe}$ metal for $\mathrm{Mn}$, and pure metal for each $\mathrm{Cr}$, and $\mathrm{Ni}$. For corundum analysis, except for magnetite that was used for $\mathrm{Fe}$, the same standards were employed. Oxides of the High Field Strength Elements (HFSE) Nb, Ta, Th, and U were analyzed at the same probe running conditions. Niobium, Ce, Y, Ta, and $\mathrm{Zr}$ were analyzed on the $\mathrm{L} \alpha$ lines. The $\mathrm{M} \alpha$ lines were used to measure Th, $\mathrm{U}$, and $\mathrm{Pb}$. Standards for the HFSE and REE are pure metal for $\mathrm{Nb}$ and $\mathrm{Ta}$, synthetic phosphates $\mathrm{CePO}_{4}, \mathrm{LaPO}_{4}$, and $\mathrm{YPO}_{4}$ for $\mathrm{Ce}, \mathrm{La}$, and $\mathrm{Y}$, zircon for $\mathrm{Zr}$, natural apatite for $\mathrm{P}$ and natural $\mathrm{UO}_{2}, \mathrm{ThO}_{2}$, and $\mathrm{PbO}$ for $\mathrm{U}$, $\mathrm{Th}$, and $\mathrm{Pb}$. Matrix corrections were done online using the ZAF correction routine.

\section{XRF}

The sapphire crystals were still inside their host rocks, offering the opportunity to classify the basalts that carried them to the surface. After removal of visible alteration rims, around $100 \mathrm{~g}$ of basaltic material was crushed and ground in an agate mill. Major elements were analyzed from fused lithium fluoride-based glass disks using a PANalytical Axios wavelength dispersive XRF.

\section{LA-ICP-MS}

Trace elements in the sapphires were measured using a Resonetics Resolution M50E $193 \mathrm{~nm}$ laser ablation system coupled to a Thermo Scientific X Series II quadrupole inductively coupled plasma mass spectrometer (Q-ICPMS). The laser was operated at a repetition rate of $15 \mathrm{~Hz}$ and a fluence of $7 \mathrm{~J} / \mathrm{cm}^{2}$. Spot sizes were $100 \mu \mathrm{m}$ in diameter, and 20-30 spots were quantified depending on grain size and homogeneity of the sapphire. Count rates were normalized using ${ }^{27} \mathrm{Al}$ as the internal standard, assuming an average of $98.5 \mathrm{wt} \% \mathrm{Al}_{2} \mathrm{O}_{3}$ as based on the EPMA analyses. The NIST-SRM-610 and NIST-SRM-612 standard reference materials were used for calibration as external standards using the preferred reference values given by Jochum et al. (2011). Isotopes monitored were ${ }^{9} \mathrm{Be},{ }^{23} \mathrm{Na},{ }^{24} \mathrm{Mg}$, ${ }^{25} \mathrm{Mg},{ }^{39} \mathrm{~K},{ }^{47} \mathrm{Ti},{ }^{51} \mathrm{~V},{ }^{52} \mathrm{Cr},{ }^{53} \mathrm{Cr},{ }^{55} \mathrm{Mn},{ }^{57} \mathrm{Fe},{ }^{66} \mathrm{Zn},{ }^{68} \mathrm{Zn},{ }^{69} \mathrm{Ga}$, ${ }^{88} \mathrm{Sr},{ }^{89} \mathrm{Y},{ }^{90} \mathrm{Zr},{ }^{93} \mathrm{Nb},{ }^{118} \mathrm{Sn},{ }^{120} \mathrm{Sn},{ }^{178} \mathrm{Hf},{ }^{180} \mathrm{Hf},{ }^{181} \mathrm{Ta},{ }^{182} \mathrm{~W}$, ${ }^{184} \mathrm{~W},{ }^{186} \mathrm{~W},{ }^{232} \mathrm{Th}$, and ${ }^{238} \mathrm{U}$. The doubly charged cation production rate monitored with ${ }^{137} \mathrm{Ba}^{++} /{ }^{137} \mathrm{Ba}$ was kept below $2 \%$.

One Columbite Group mineral inclusion was dated on the basis of the U-Pb decay system, using in situ LAICP-MS analysis. This inclusion was analyzed for $\mathrm{U}, \mathrm{Th}$, and $\mathrm{Pb}$ isotopes by LA-ICP-MS at the Institute of Geosciences, Johann Wolfgang Goethe University Frankfurt, using a Thermo Scientific Element II sector field ICPMS coupled to a New Wave Research UP-213 ultraviolet laser system. Data were acquired in the analog mode for ${ }^{238} \mathrm{U}$ and in the counting mode for all other isotopes with $20 \mathrm{~s}$ measurement on the background followed by sample ablation of $20 \mathrm{~s}$. Prior to analysis, the sample surface was cleaned with 3 pulses of pre-ablation. Square laser spots with an edge length of $28 \mu \mathrm{m}$ were used. The laser was operated at a repetition rate of $6 \mathrm{~Hz}$ and an energy fluence of $2 \mathrm{~J} / \mathrm{cm}^{2}$ with an energy output of $50 \%$. The signal was tuned to obtain maximum sensitivity for ${ }^{238} \mathrm{U}$ and ${ }^{206} \mathrm{~Pb}$, and to keep the oxide production monitored as ${ }^{254} \mathrm{UO} /{ }^{238} \mathrm{U}$ below $3 \%$. Measured masses were ${ }^{202} \mathrm{Hg},{ }^{204} \mathrm{Hg},{ }^{204} \mathrm{~Pb},{ }^{206} \mathrm{~Pb},{ }^{207} \mathrm{~Pb},{ }^{208} \mathrm{~Pb},{ }^{232} \mathrm{Th},{ }^{235} \mathrm{U}$, and ${ }^{238} \mathrm{U}$. Mercury was also measured because ${ }^{204} \mathrm{Hg}$ can be a minor contaminant of the Ar carrier gas, potentially causing isobaric interferences on ${ }^{204} \mathrm{~Pb}$.

The primary standard used was the GJ-1 zircon, reported by Jackson et al. (2004) to have a TIMS age of $608 \pm 0.4 \mathrm{Ma}$. Isotopic ratios were corrected for ablationinduced elemental fractionation offset between zircon matrix and columbite offset between zircon matrix and columbite matrix using a columbite-tantalite solid solution (Coltan 139) from Madagascar that is being used as an in-house standard for coltan LA-ICP-MS analyses by the Federal Institute for Geosciences and Natural Resources, Germany (Gäbler et al. 2011). The usability of Coltan 139 as a matrix matching standard for Columbite Group Mineral LA-ICPMS U-Pb dating was recently demonstrated by Che et al. (2015). Coltan 007 from Kokobin, Ghana (Melcher et al. 2008, 2015) was used as a secondary standard to control the accuracy and reproducibility of the measurements. For Coltan 139, Melcher et al. (2015) reported TIMS ages of $505.4 \pm 1.0 \mathrm{Ma}$ (BGR, Hannover) and $506.6 \pm 2.4 \mathrm{Ma}$ (University of Toronto), and an LA-ICP-MS age of $506.2 \pm 5.0 \mathrm{Ma}$ (University of Frankfurt). Coltan 007 has an age of 2079.6 $\pm 3.1 \mathrm{Ma}$ (Melcher et al. 2008, 2015). 
A total number of 45 unknowns were analyzed, using the standard bracketing method. Acquired data were then processed using an in-house MS Excel@ spreadsheet (Gerdes and Zeh 2006, 2009). A ${ }^{208} \mathrm{~Pb}$-based common lead correction was applied, as there is very low Th in the sample.

\section{Raman spectroscopy}

Raman spectroscopy was used to further characterize all types of inclusions in the sapphire, and special attention was paid to those that are not exposed on the polished surface, including solid inclusions as well as fluid inclusions and their daughter minerals. As fluid inclusions were not recognizable in polished sapphire mounts, for the Raman analyses, polished thick sections with a thickness of $\sim 500 \mu \mathrm{m}$ were prepared, so that the sapphires were translucent. Unfortunately, some sapphires were too brittle for thick sectioning. Because of this, we cannot say for certain that all the sapphires depicted here have inclusions, or whether the inclusions shown here are representative of all SVF sapphires.

Raman spectroscopy was also employed to distinguish between calcium carbonate polymorphs in carbonate inclusions. Raman spectra were acquired with a confocal Horiba HR800 equipped with an Olympus BX41 optical microscope operated in $180^{\circ}$ backscatter geometry. The excitation source was an Nd-YAG Laser with a wave length of $532.18 \mathrm{~nm}$. The laser beam was focused on the target with a $\times 50$ objective lense. Spectra were accumulated with an exposure time of $30 \mathrm{~s}$ on each point within a frequency range of $100-4000 \mathrm{~cm}^{-1}$. The laser power was $200 \mathrm{~mW}$.

\section{TEM}

One sapphire (sample PE25) was investigated with the transmission electron microscope (TEM) at the GeoForschungsZentrum (GFZ) in Potsdam, Germany. Next to the typical blue colored areas, this sapphire has a gray to brown colored domain (Fig. 2) that is characterized by a significant enrichment in the HFSE up to two orders of magnitude higher than the HFSE content in the blue domains (up to $5000 \mathrm{ppm} \mathrm{Nb}$ in the brownish domain). Given that some sapphire domains contain up to $5000 \mathrm{ppm}$ HFSE, it would be interesting to determine whether these elements substitute into the corundum crystal lattice, or whether they are present as nano-inclusions. Therefore, a combination of the focused ion beam (FIB) and the TEM techniques was applied to retrieve information about the crystallographic orientation relations of the sapphire and any potential nano-inclusions at an atomic scale, revealing the physicochemical state of the HFSE in the sapphires. For this purpose, three electron-transparent foils $(15 \times 10 \times 0.15 \mu \mathrm{m})$ - two from the brownish areas, and one from the blue area-were cut, using the focused ion beam (FIB) technique (Ohnishi et al. 1990; Ishitani et al. 1990) where thin membranes of an area of interest are cut from a polished sample by sputtering with $\mathrm{Ga}^{+}$ions accelerated to $30 \mathrm{keV}$. The FIB sections from the brownish area were cut into two different crystallographic orientations, one near parallel to [0001] and another one inclined to [0001] of the sapphire. The section of the blue area was cut near parallel to [0001]. Subsequently, the FIB sections were thinned with a $\mathrm{Ga}$ ion beam to a final thickness of $25-30 \mathrm{~nm}$ to guarantee electron transparency. A detailed description of the principles of the FIB technique is given by Wirth $(2004,2009)$. The FIB sections were subsequently analyzed with an FEI Tecnai F20 X-Twin TEM with a Schottky field emitter as electron source. The instrument is equipped with a Fishione high-angle annular darkfield (HAADF) detector allowing for Z-contrast sensitive imaging, an EDAX energy dispersive $X$-ray spectroscopic system (EDS) for the identification of nano-scale chemical compositions from the excited characteristic X-rays of the sample, as well as a Gatan Tridiem energy filter and an electron energy loss spectrometer (EELS) for spectrum imaging.

\section{Results}

\section{Host rock composition and corundum mineral chemistry}

Host rock compositions and their CIPW norms are presented in Table 2. All rocks are nepheline normative, though to various extends, ranging from 0.3 to $10 \mathrm{wt} \%$ normative nepheline. In the Total Alkali vs. Silica (TAS) diagram (Le Maitre 2002), most host rocks plot in the field for alkali basalts. One sapphire was entrained in a basanite, and three samples lie in the trachybasalt (hawaiite) field.

The Siebengebirge sapphires include up to $2 \mathrm{wt} \% \mathrm{FeO}$ and up to $0.8 \mathrm{wt} \% \mathrm{TiO}_{2}$. The major and trace element compositions of the sapphires, as well as the major element composition of the spinel coronas, are listed in Table 3.

Next to $\mathrm{Fe}$ and $\mathrm{Ti}$ as the main impurities, all sapphires contain detectable amounts of $\mathrm{Mg}, \mathrm{Mn}, \mathrm{Ga}, \mathrm{V}, \mathrm{Nb}, \mathrm{W}$, and Th. Beryllium, Na, K, Cr, Zn, Zr, Ta, and Hf were detected in few analyses, whereas $\mathrm{Sr}, \mathrm{Y}$, and $\mathrm{U}$ are usually below detection limit. Remarkably, the highest concentrations of the HFSE (up to $5000 \mathrm{ppm} \mathrm{Nb}$ and up to $2900 \mathrm{ppm} \mathrm{Ta}$ ), as well as of Th (up to $226 \mathrm{ppm}$ ) were detected in the brown to gray domains in samples PE21 and PE25 where the concentrations are up to two orders of magnitude higher than in the blue domains of the same sapphire. The other elements are not concentrated at any preferred site within the sapphire. The Ga contents range from 48 to $256 \mathrm{ppm}$. 
Table 2 XRF analyses of the host rocks of the Siebengebirge sapphires

\begin{tabular}{|c|c|c|c|c|c|c|c|c|c|c|c|c|c|c|}
\hline & 3 & 15 & 26 & \multicolumn{2}{|c|}{ KS 21} & OL 25 & OL 60 & \multicolumn{2}{|l|}{ OL 61} & \multicolumn{2}{|l|}{ UN 31} & \multicolumn{2}{|l|}{ PE 21} & PE 25 \\
\hline $\mathrm{SiO}_{2}$ & 43.7 & 46.1 & 45.4 & \multicolumn{2}{|c|}{46.5} & 46.9 & 46.3 & \multicolumn{2}{|l|}{46.0} & \multicolumn{2}{|l|}{46.2} & \multicolumn{2}{|l|}{46.2} & 46.6 \\
\hline $\mathrm{Al}_{2} \mathrm{O}_{3}$ & 12.6 & 14.3 & 13.8 & \multicolumn{2}{|c|}{14.8} & 14.2 & 14.3 & \multicolumn{2}{|l|}{14.2} & 14.2 & & \multicolumn{2}{|l|}{13.9} & 14.0 \\
\hline $\mathrm{Fe}_{2} \mathrm{O}_{3}$ & 12.0 & 11.4 & 11.3 & \multicolumn{2}{|c|}{11.5} & 11.2 & 11.4 & \multicolumn{2}{|l|}{11.1} & 11.4 & & \multicolumn{2}{|l|}{12.4} & 12.4 \\
\hline $\mathrm{MnO}$ & 0.19 & 0.18 & 0.20 & \multicolumn{2}{|c|}{0.19} & 0.18 & 0.18 & \multicolumn{2}{|l|}{0.18} & 0.18 & & \multicolumn{2}{|l|}{0.20} & 0.19 \\
\hline $\mathrm{MgO}$ & 12.1 & 9.41 & 9.72 & \multicolumn{2}{|c|}{8.01} & 8.68 & 9.07 & \multicolumn{2}{|l|}{9.24} & 9.27 & & \multicolumn{2}{|l|}{9.09} & 8.92 \\
\hline $\mathrm{CaO}$ & 10.7 & 9.84 & 10.6 & \multicolumn{2}{|c|}{9.62} & 10.1 & 9.87 & \multicolumn{2}{|l|}{9.80} & 9.87 & & 9.32 & & 9.40 \\
\hline $\mathrm{Na}_{2} \mathrm{O}$ & 3.10 & 3.35 & 3.03 & 2. & 75 & 3.60 & 3.80 & 3.66 & & 3.58 & & 3.05 & & 3.26 \\
\hline $\mathrm{K}_{2} \mathrm{O}$ & 1.21 & 1.38 & 1.37 & 1. & 37 & 1.40 & 1.44 & 1.43 & & 1.35 & & 1.15 & & 1.19 \\
\hline $\mathrm{TiO}_{2}$ & 2.28 & 2.23 & 2.21 & 2. & 35 & 2.25 & 2.24 & 2.23 & & 2.26 & & 2.19 & & 2.20 \\
\hline $\mathrm{P}_{2} \mathrm{O}_{5}$ & 0.55 & 0.46 & 0.52 & 0. & 41 & 0.46 & 0.45 & 0.45 & & 0.46 & & 0.45 & & 0.44 \\
\hline $\mathrm{SO}_{3}$ & 0.16 & 0.17 & 0.10 & 0 . & 15 & 0.13 & 0.18 & 0.07 & & 0.15 & & 0.17 & & 0.19 \\
\hline L.O.I. & 0.45 & 0.73 & 0.62 & 1. & 34 & 0.16 & 0.53 & 0.47 & & 0.63 & & 0.77 & & 0.90 \\
\hline Sum & 99.0 & 99.6 & 98.9 & 99 & .0 & 99.3 & 99.8 & 98.8 & & 99.6 & & 98.9 & & 99.7 \\
\hline Rock type & Basanite & $\begin{array}{l}\text { Alkali } \\
\text { Basalt }\end{array}$ & $\begin{array}{l}\text { Alkali } \\
\text { Basalt }\end{array}$ & & $\begin{array}{l}\text { Alkali } \\
\text { Basalt }\end{array}$ & Hawaiite & Hawaiite & Hawaiite & $\begin{array}{l}\text { Alkali } \\
\text { Basalt }\end{array}$ & & $\begin{array}{l}\text { Alkali } \\
\text { Basalt }\end{array}$ & & Alkali & Basalt \\
\hline CIPW norm & & & & & & & & & & & & & & \\
\hline Plagioclase & 25.0 & 37.9 & 34.7 & & 48.1 & 37.4 & 34.5 & 34.7 & 36.5 & & 35.0 & & 42.9 & \\
\hline Orthoclase & 7.33 & 8.33 & 8.33 & & 8.39 & 8.45 & 8.69 & 8.69 & 8.16 & & 8.1 & & 7.21 & \\
\hline Nepheline & 10.1 & 5.9 & 6.46 & & 0.21 & 6.54 & 8.49 & 8.4 & 7.08 & & 7.21 & & 2.82 & \\
\hline Diopside & 26.7 & 21.0 & 24.1 & & 17.4 & 23.4 & 22.9 & 22.8 & 22.2 & & 22.8 & & 19.2 & \\
\hline Olivine & 23.0 & 19.4 & 19.0 & & 18.4 & 16.9 & 18.0 & 18.2 & 18.7 & & 19.3 & & 20.3 & \\
\hline Ilmenite & 4.44 & 4.33 & 4.31 & & 4.62 & 4.35 & 4.33 & 4.35 & 4.39 & & 4.35 & & 4.27 & \\
\hline Magnetite & 1.78 & 1.70 & 1.68 & & 1.73 & 1.65 & 1.68 & 1.65 & 1.68 & & 1.83 & & 1.84 & \\
\hline Apatite & 1.32 & 1.09 & 1.23 & & 0.97 & 1.09 & 1.07 & 1.07 & 1.09 & & 1.07 & & 1.04 & \\
\hline $\mathrm{Na}_{2} \mathrm{SO}_{4}$ & 0.31 & 0.3 & 0.18 & & 0.28 & 0.23 & 0.32 & 0.12 & 0.27 & & 0.32 & & 0.35 & \\
\hline Total & 99.9 & 100.0 & & 100.0 & 100.0 & 100.0 & 100.0 & 100.0 & 100.0 & & 100.0 & & 100.0 & \\
\hline
\end{tabular}

The CIPW norm was calculated on the assumption that the $\mathrm{Fe}^{3+/} \mathrm{Fe}_{\text {tot }}$ ratio of the basalt is 0.1

In terms of their $\mathrm{Ga} / \mathrm{Mg}$ ratio and the total $\mathrm{Fe}$ concentration, the Siebengebirge sapphires can be divided into two groups: (1) Sapphires with low $\mathrm{Ga} / \mathrm{Mg}(<10)$ and high $\mathrm{Fe}_{\text {tot }}$ and (2) sapphires with high $\mathrm{Ga} / \mathrm{Mg}(>10)$ ratios and lower $\mathrm{Fe}_{\text {tot}}$.

\section{Inclusions in sapphire}

Inclusions have high potential for providing important information on the origin of sapphires. If the inclusions are primary, i.e., crystallized during growth of the sapphires, then they may represent melts, fluids, or accessory minerals that coexisted with the sapphires during crystal growth. Secondary inclusions may provide information about a potential post-magmatic alteration of the sapphires. Inclusions are interpreted to be primary, if they are completely enclosed within the sapphire and are not located at any visible cracks. An inclusion has to be regarded to be of secondary origin if it is xenomorphic and restricted to cracks in the sapphire.

The Siebengebirge sapphires contain a broad spectrum of mineral inclusions, including silicates, carbonates, and a wide variety of $\mathrm{Nb}$, $\mathrm{Ta}$ and $\mathrm{Th}$ oxides. While some sapphires are virtually inclusion free, others have a high abundance and compositional variability among the mineral inclusions. Notably, there is no correlation between the mineral inclusion suite and the minor and trace element compositions of the host sapphires. Interestingly, many inclusions in the sapphires are carbonates, which have only been described previously in sapphires associated with alkaline basaltic volcanism from the Bo Phloi gemfield in Kanchanaburi, western Thailand (Khamloet et al. 2014). Furthermore, the U-bearing inclusions thorite, thorianite and Pyrochlore and Columbite Group minerals are of great interest, as they potentially allow to date the age of the sapphire crystallization using the $\mathrm{U}-\mathrm{Pb}$ chronometer. With 
Table 3 Major element (EPMA) and trace element (LA-ICP-MS) composition of the Siebengebirge sapphires and spinel coronas

\begin{tabular}{|c|c|c|c|c|c|c|c|c|c|c|c|c|c|}
\hline & \multicolumn{2}{|l|}{3} & \multicolumn{3}{|l|}{15} & \multicolumn{2}{|l|}{26} & \multicolumn{2}{|l|}{$\mathrm{KS} 21$} & \multicolumn{2}{|l|}{ OL25 } & \multicolumn{2}{|l|}{ OL60 } \\
\hline & Crn & Spl & $\mathrm{Crn}$ & & Spl & Crn & Spl & Crn & Spl & Crn & Spl & Crn & Spl \\
\hline \multicolumn{14}{|l|}{ wt $\%$} \\
\hline $\mathrm{SiO}_{2}$ & 0.02 & 0.03 & 0.0 & & 0.1 & 0.02 & 0.02 & 0.01 & 0.02 & n.a. & bdl & 0.02 & 0.02 \\
\hline $\mathrm{TiO}_{2}$ & 0.10 & 0.11 & 0.1 & & 0.09 & 0.12 & 0.07 & 0.18 & 0.23 & 0.11 & 0.12 & 0.06 & 0.08 \\
\hline $\mathrm{Al}_{2} \mathrm{O}_{3}$ & 97.6 & 65.4 & 98. & & 65.6 & 99.5 & 65.6 & 98.6 & 64.8 & 99.4 & 65.5 & 99.7 & 65.4 \\
\hline $\mathrm{FeO}$ & 1.17 & 16.8 & 2.0 & & 18.5 & 0.87 & 18.9 & 1.52 & 21.1 & 1.57 & 20.6 & 0.43 & 17.8 \\
\hline $\mathrm{MnO}$ & 0.01 & 0.27 & 0.0 & & 0.28 & 0.02 & 0.3 & 0.01 & 0.27 & 0.01 & 0.33 & bdl & 0.24 \\
\hline $\mathrm{MgO}$ & bdl & 16.4 & 0.0 & & 16.1 & bdl & 15.8 & bdl & 14.2 & bdl & 15.2 & bdl & 16.7 \\
\hline $\mathrm{CaO}$ & bdl & 0.02 & bdl & & 0.02 & 0.01 & 0.01 & 0.02 & 0.04 & bdl & bdl & 0.01 & 0.01 \\
\hline $\mathrm{Na}_{2} \mathrm{O}$ & bdl & bdl & 0.0 & & 0.02 & 0.01 & 0.01 & 0.02 & bdl & bdl & bdl & 0.03 & 0.01 \\
\hline $\mathrm{K}_{2} \mathrm{O}$ & bdl & bdl & bdl & & 0.01 & 0.01 & bdl & bdl & bdl & bdl & n.a. & 0.01 & bdl \\
\hline $\mathrm{Cr}_{2} \mathrm{O}_{3}$ & bdl & 0.03 & bdl & & 0.02 & bdl & 0.01 & 0.01 & 0.01 & 0.01 & 0.01 & 0.02 & 0.01 \\
\hline Total & 98.9 & 99.0 & 100 & & 100.6 & 100.6 & 100.7 & 100.4 & 100.6 & 101.1 & 101.7 & 100.2 & 100.3 \\
\hline$n$ & 3 & 4 & 3 & & 5 & 3 & 7 & 2 & 3 & 20 & 10 & 3 & 5 \\
\hline Spinel & & 63 & & & 60 & & 60 & & 55 & & 57 & & 64 \\
\hline Hercynite & & 37 & & & 40 & & 40 & & 45 & & 43 & & 36 \\
\hline ppm & $(n=32)$ & & \multicolumn{3}{|c|}{$(n=30)$} & \multicolumn{2}{|l|}{$(n=31)$} & \multicolumn{2}{|l|}{$(n=32)$} & \multicolumn{2}{|l|}{$(n=30)$} & \multicolumn{2}{|l|}{$(n=16)$} \\
\hline $\mathrm{Na}$ & 15-198 & & \multicolumn{3}{|c|}{$4-144$} & \multicolumn{2}{|l|}{$8-373$} & \multicolumn{2}{|l|}{ bdl-144 } & \multicolumn{2}{|l|}{ bdl-359 } & \multicolumn{2}{|l|}{ 8-1007 } \\
\hline $\mathrm{K}$ & $6-94$ & & \multicolumn{3}{|c|}{$10-1152$} & \multicolumn{2}{|l|}{$12-283$} & bdl & & bdl-222 & & $14-39$ & \\
\hline $\mathrm{Be}$ & $0.06-2.3$ & & 0.0 & & & $0.1-2$ & & bdl-3 & & bdl-2 & & $0.3-26$ & \\
\hline $\mathrm{Mg}$ & $22-10,803$ & & $39-$ & & & $5-253$ & & $4-39$ & & $7-90$ & & $2-860$ & \\
\hline $\mathrm{Sr}$ & bdl & & bdl & & & bdl & & bdl & & bdl & & bdl & \\
\hline $\mathrm{Ti}$ & $158-2880$ & & 171 & 1182 & & $78-1292$ & & $158-1316$ & & $76-5154$ & & $60-659$ & \\
\hline V & $8-15$ & & $7-1$ & & & $2-12$ & & $3-15$ & & $5-19$ & & $2-12$ & \\
\hline $\mathrm{Cr}$ & bdl & & $1-1$ & & & $2-8$ & & bdl-2 & & bdl-2 & & $0.8-2$ & \\
\hline Mn & $1.2-212$ & & $2-2$ & & & 4-92 & & $1-13$ & & bdl-49 & & $3-18$ & \\
\hline $\mathrm{Fe}$ & 5559-18295 & & 856 & $-14,958$ & & 2967-16, & & $4599-15,967$ & & $3177-12,122$ & & $1875-8549$ & \\
\hline $\mathrm{Zn}$ & $1.9-212$ & & $2-1$ & & & $2-9$ & & bdl-714 & & bdl-10 & & $2-11$ & \\
\hline $\mathrm{Ga}$ & $127-238$ & & $91-$ & & & 149-256 & & $135-252$ & & $87-147$ & & $135-210$ & \\
\hline Sn & $0.13-9$ & & 0.1 & & & $0.1-2$ & & $0.2-20$ & & $0.1-2$ & & bdl-10 & \\
\hline $\mathrm{Y}$ & bdl & & bdl & & & bdl & & bdl & & bdl -0.2 & & bdl & \\
\hline $\mathrm{Zr}$ & $0.1-1.94$ & & bdl & & & bdl & & bdl-4 & & bdl- -0.6 & & bdl & \\
\hline $\mathrm{Nb}$ & $0.75-706$ & & 0.2 & & & $0.5-710$ & & bdl-806 & & $0.6-96$ & & $0.3-814$ & \\
\hline $\mathrm{Ta}$ & $0.43-362$ & & bdl & 6.4 & & $0.1-25$ & & $0.03-342$ & & bdl-46 & & $0.2-370$ & \\
\hline ppb & & & & & & & & & & & & & \\
\hline Hf & $10-600$ & & 10 & & & 10-130 & & bdl-600 & & bdl- -0.1 & & bdl-200 & \\
\hline W & 10-290 & & 10 & & & $10-560$ & & bdl-1735 & & bdl-750 & & bdl-600 & \\
\hline Th & $10-7030$ & & 10 & & & bdl-890 & & bdl-14 (ppm) & & $10-5310$ & & 10-9960 & \\
\hline $\mathrm{U}$ & bdl-10 & & bdl & & & bdl-470 & & bdl-20 & & bdl-120 & & bdl-20 & \\
\hline & Ratios & $2 \sigma$ & & tios & $2 \sigma$ & Ratios & $2 \sigma$ & Ratios & $2 \sigma$ & Ratios & $2 \sigma$ & Ratios & $2 \sigma$ \\
\hline $\mathrm{Ga} / \mathrm{Mg}$ & 3.6 & 4.1 & & & 0.8 & 17.1 & 16.2 & 16.0 & 31.4 & 6.3 & 7.9 & 23.4 & 57.8 \\
\hline & OL61 & & & UN31 & & & PE21 & & PE25 & & & 52 & \\
\hline & Crn & & Spl & Crn & & Spl & Crn & Spl & Crn & Spl & & Crn & Spl \\
\hline $\mathrm{wt} \%$ & & & & & & & & & & & & & \\
\hline $\mathrm{SiO}_{2}$ & 0.02 & & 0.01 & 0.01 & & 0.01 & 0.02 & 0.01 & 0.02 & 0.05 & & 0.02 & 0.02 \\
\hline $\mathrm{TiO}_{2}$ & 0.03 & & 0.1 & 0.2 & & 0.14 & 0.75 & 0.27 & 0.25 & 0.15 & & .1 & 0.08 \\
\hline
\end{tabular}


Table 3 continued

\begin{tabular}{|c|c|c|c|c|c|c|c|c|c|c|}
\hline & \multicolumn{2}{|l|}{ OL61 } & \multicolumn{2}{|l|}{ UN31 } & \multicolumn{2}{|l|}{ PE21 } & \multicolumn{2}{|l|}{ PE25 } & \multicolumn{2}{|l|}{ S52 } \\
\hline & $\mathrm{Crn}$ & Spl & $\mathrm{Crn}$ & Spl & Crn & Spl & $\mathrm{Crn}$ & Spl & $\mathrm{Crn}$ & Spl \\
\hline $\mathrm{Al}_{2} \mathrm{O}_{3}$ & 97.8 & 64.7 & 98.2 & 65.0 & 98.1 & 63.7 & 99.4 & 65.1 & 97.8 & 65.1 \\
\hline $\mathrm{FeO}$ & 1.31 & 17.0 & 1.46 & 18.8 & 0.86 & 20.7 & 0.92 & 21.3 & 1.67 & 18.2 \\
\hline $\mathrm{MnO}$ & 0.02 & 0.21 & bdl & 0.26 & 0.01 & 0.26 & 0.02 & 0.3 & bdl & 0.28 \\
\hline $\mathrm{MgO}$ & 0.01 & 16.1 & 0.01 & 15.6 & bdl & 14.2 & 0.01 & 14.1 & 0.01 & 15.9 \\
\hline $\mathrm{CaO}$ & 0.01 & 0.01 & 0.01 & 0.01 & 0.01 & 0.03 & 0.01 & 0.02 & 0.01 & 0.02 \\
\hline $\mathrm{Na}_{2} \mathrm{O}$ & 0.01 & 0.01 & bdl & 0.01 & 0.01 & bdl & 0.01 & 0.02 & bdl & 0.02 \\
\hline $\mathrm{K}_{2} \mathrm{O}$ & 0.01 & bdl & bdl & bdl & 0.01 & 0.01 & 0.01 & 0.01 & 0.01 & bdl \\
\hline $\mathrm{Cr}_{2} \mathrm{O}_{3}$ & 0.01 & 0.01 & bdl & bdl & n.d. & 0.02 & 0.02 & 0.02 & 0.01 & 0.01 \\
\hline Total & 99.2 & 98.1 & 99.9 & 99.8 & 99.8 & 99.2 & 100.7 & 101.0 & 99.7 & 99.6 \\
\hline$n$ & 2 & 3 & 3 & 4 & 3 & 5 & 3 & 2 & 3 & 5 \\
\hline Spinel & & 63 & & 60 & & 56 & & 54 & & 61 \\
\hline Hercynite & & 37 & & 40 & & 44 & & 46 & & 39 \\
\hline ppm & $(n=30)$ & & $(n=30)$ & & $(n=30)$ & & $(n=36)$ & & $(n=31)$ & \\
\hline $\mathrm{Na}$ & bdl-469 & & bdl-60 & & bdl-79 & & bdl-149 & & bdl-1644 & \\
\hline $\mathrm{K}$ & bdl-732 & & bdl & & bdl-52 & & bdl-109 & & bdl-427 & \\
\hline $\mathrm{Be}$ & bdl-0.7 & & bdl-1 & & bdl-10 & & $0.7-11$ & & bdl-0.8 & \\
\hline $\mathrm{Mg}$ & $10-45$ & & $61-174$ & & $10-34$ & & $13-243$ & & $42-152$ & \\
\hline $\mathrm{Sr}$ & bdl-8 & & bdl & & bdl- -0.2 & & bdl- -0.2 & & bdl-11 & \\
\hline $\mathrm{Ti}$ & $75-1205$ & & $641-2149$ & & $1045-6231$ & & $876-6226$ & & $150-490$ & \\
\hline V & $6-10$ & & $28-66$ & & $13-37$ & & $12-29$ & & $6-12$ & \\
\hline $\mathrm{Cr}$ & bdl-4 & & bdl-22 & & bdl-20 & & bdl-21 & & bdl-5 & \\
\hline $\mathrm{Mn}$ & bdl-10 & & bdl-3.7 & & bdl-196 & & bdl-5 & & bdl-33 & \\
\hline $\mathrm{Fe}$ & $4598-10,728$ & & $6545-12,701$ & & $3301-6693$ & & $3853-6168$ & & $6519-10,434$ & \\
\hline $\mathrm{Zn}$ & $2-14$ & & $2-11$ & & bdl-5 & & bdl-7 & & bdl-5 & \\
\hline $\mathrm{Ga}$ & $107-167$ & & $133-205$ & & 113-193 & & 116-188 & & $48-90$ & \\
\hline $\mathrm{Sn}$ & bdl-0.6 & & $0.3-9$ & & $2-21$ & & $0.4-14$ & & bdl-0.1 & \\
\hline Y & bdl & & bdl & & bdl- -0.5 & & bdl- -0.5 & & bdl-4 & \\
\hline $\mathrm{Zr}$ & bdl-0.6 & & bdl-3 & & $0.4-22$ & & $0.3-22$ & & bdl-1 & \\
\hline $\mathrm{Nb}$ & bdl-9 & & bdl-748 & & 3-3998 & & $12-2771$ & & bdl-18 & \\
\hline $\mathrm{Ta}$ & $0.1-4$ & & $0.1-169$ & & $4-1699$ & & $8-1011$ & & bdl-1992 & \\
\hline \multicolumn{11}{|l|}{$\mathrm{ppb}$} \\
\hline Hf & bdl-35 & & bdl-552 & & $113-4825$ & & $33-4085$ & & bdl-14 & \\
\hline W & bdl-376 & & bdl-1770 & & 995-41 (ppm) & & $117-23(\mathrm{ppm})$ & & bdl-388 & \\
\hline $\mathrm{Th}$ & bdl-74 & & bdl-21.3 (ppm) & & $1-166$ (ppm) & & $0.6-226(\mathrm{ppm})$ & & bdl-3264 & \\
\hline \multirow[t]{2}{*}{$\mathrm{U}$} & bdl-24 & & bdl-40 & & bdl-419 & & bdl-0.6 & & bdl-741 & \\
\hline & Ratios & $2 \sigma$ & Ratios & $2 \sigma$ & Ratios & $2 \sigma$ & Ratios & $2 \sigma$ & Ratios & $2 \sigma$ \\
\hline $\mathrm{Ga} / \mathrm{Mg}$ & 1.3 & 2.0 & 1.6 & 0.7 & 5.8 & 3.3 & 6.6 & 4.1 & 1.0 & 0.3 \\
\hline
\end{tabular}

such age information, the sapphires may be temporally related with to their host basalts, as the age of the basaltic volcanism in the SVF is well known (Todt and Lippolt 1980; Linthout et al. 2009; Przybyla 2013).

\section{Silicates}

Silicate mineral inclusions are potassic feldspar and labradoritic plagioclase. In one sample (OL25), feldspar was found associated with calcite, either as discrete mineral grains attached to calcite or as a thin rim around the carbonate phase. These associations are interpreted to be primary. In sample 26, the feldspars are located in vesicles along cracks within sapphire and associated with clay minerals and zeolites; hence, these are considered to be secondary in origin. This population of silicate mineral inclusions may not be representative for all investigated sapphires, as only few polished surfaces were studied. Yet, it is striking that 
Fig. 4 Backscattered electron images of selected carbonate inclusions in some Siebengebirge sapphires. a Calcite inclusion in sample 3. b Dolomite inclusion in sample 26. c Vesicular secondary aragonite in alkali feldspar located at cracks in sample 26. d Spherical calcite inclusions in phonolitic silicate glass in sample 26 .

Mineral abbreviations are after Whitney and Evans (2010)
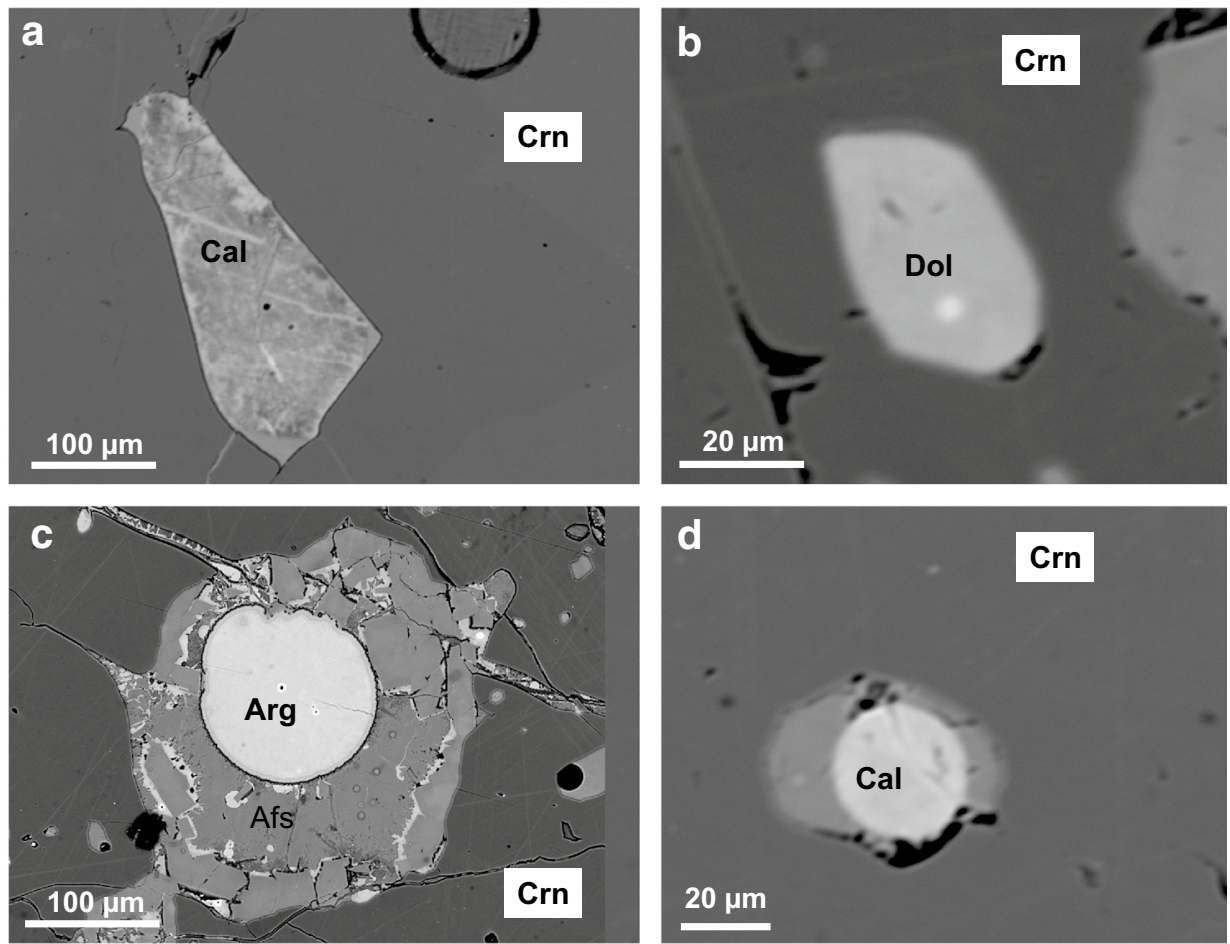

apparently only feldspar has been incorporated as a crystalline silicate mineral, but not pyroxene or olivine, which are ubiquitous liquidus phases in the host basalt samples.

\section{Carbonates}

Carbonate inclusions are observed in samples 3, 15, 26, OL25, and S52. Compositions range from calcium carbonate to magnesian carbonate. Vibrational spectroscopic studies of the carbonate inclusions in sample 26 using Raman spectroscopy revealed that these carbonates are either dolomite, calcite, or aragonite. Calcite and dolomite inclusions can be regarded as primary as these are completely included in the host sapphire. By contrast, aragonite tends to form vesicular textures within secondary potassic feldspar in the sapphire. Consequently, aragonite is considered a secondary low-temperature alteration phase. Figure 4 shows selected carbonate inclusions inside the Siebengebirge sapphires.

\section{Oxides}

Samples 3, 15, 26, and OL25 have various oxide inclusions. All oxide inclusions are completely isolated inside the sapphire, and are not located at any visible cracks (Fig. 5). Hence, they may be regarded as primary inclusions that formed cogenetically with the host sapphires. Figure 5a shows a Columbite Group $(\mathrm{Mn})(\mathrm{Fe}, \mathrm{Nb})_{2} \mathrm{O}_{6}$ inclusion that in turn includes a complex aggregate of pyrochlore $\mathrm{Ca}_{2} \mathrm{Nb}_{2} \mathrm{O}_{7}$, thorite $\mathrm{ThO}_{2}$, and thorianite $\mathrm{ThSiO}_{4}$ (Fig. 5b). Abundant base metal oxides are spinel-hercynite solid solutions $(\mathrm{Mg}, \mathrm{Fe}) \mathrm{Al}_{2} \mathrm{O}_{4}$, ilmenitepyrophanite solid solutions $(\mathrm{Fe}, \mathrm{Mn}) \mathrm{TiO}_{3}$ with exsolved hercynite and $\mathrm{Nb}$-rutile $(\mathrm{Nb}, \mathrm{Ti}, \mathrm{Fe}) \mathrm{O}_{2}$, and ilmenite $\mathrm{FeTiO}_{3}$. Figure 5c shows a complex oxide inclusion where ilmenite-pyrophanite ss showing with $\mathrm{Nb}$-rich rutile and hercynite exsolutions is rimmed by spinel at the contact to corundum, indicating a reaction of the ilmenite-pyrophanite ss with the host corundum at magmatic temperatures accounting for a primary origin. In addition, one Nb-rutile-Betafite Group $(\mathrm{Ca}, \mathrm{U})_{2}(\mathrm{Ti}, \mathrm{Nb}, \mathrm{Ta})_{2} \mathrm{O}_{6}(\mathrm{OH})$ composite aggregate was found in sample OL25. The Columbite Group inclusion from sample 26 (Fig. 5a) was used for in situ U-Pb dating using LA-ICP-MS.

\section{Melt inclusions}

Sample 26 contains composite silicate glasses of two different compositions (Fig. 6), interpreted to represent silicate melts that were trapped by the growing sapphires. One composition (melt 1) forms vesicular structures within a second glass referred to as melt 2 . Melt 1 is quite complex and heterogeneous in composition, and appears to represent a carbonated silicate melt, while melt 2 is close to an aluminous phonolite. The average compositions of the silicate glasses are compiled in Table 4. Most studies on basalt-hosted sapphires (e.g., McGee 2005; Pakhomova et al. 2006; Izokh et al. 2010; 
Fig. 5 Complex composite oxide inclusions in the Siebengebirge sapphires. $a$ and $b$ are inclusions in sample 26, c and $\mathbf{d}$ are inclusions in sample OL25. Bet Betafite Group, $\mathrm{Crn}$ corundum, $\mathrm{Col}$ Columbite Group, Herc hercynite, Ilm ilmenite, Ilme $\mathrm{Nb}$-rich rutile (ilmenorutile), $\mathrm{Pcl}$ Pyrochlore Group, Pph Pyrophanite, Spl spinel, Thr thorite, Thrn thorianite
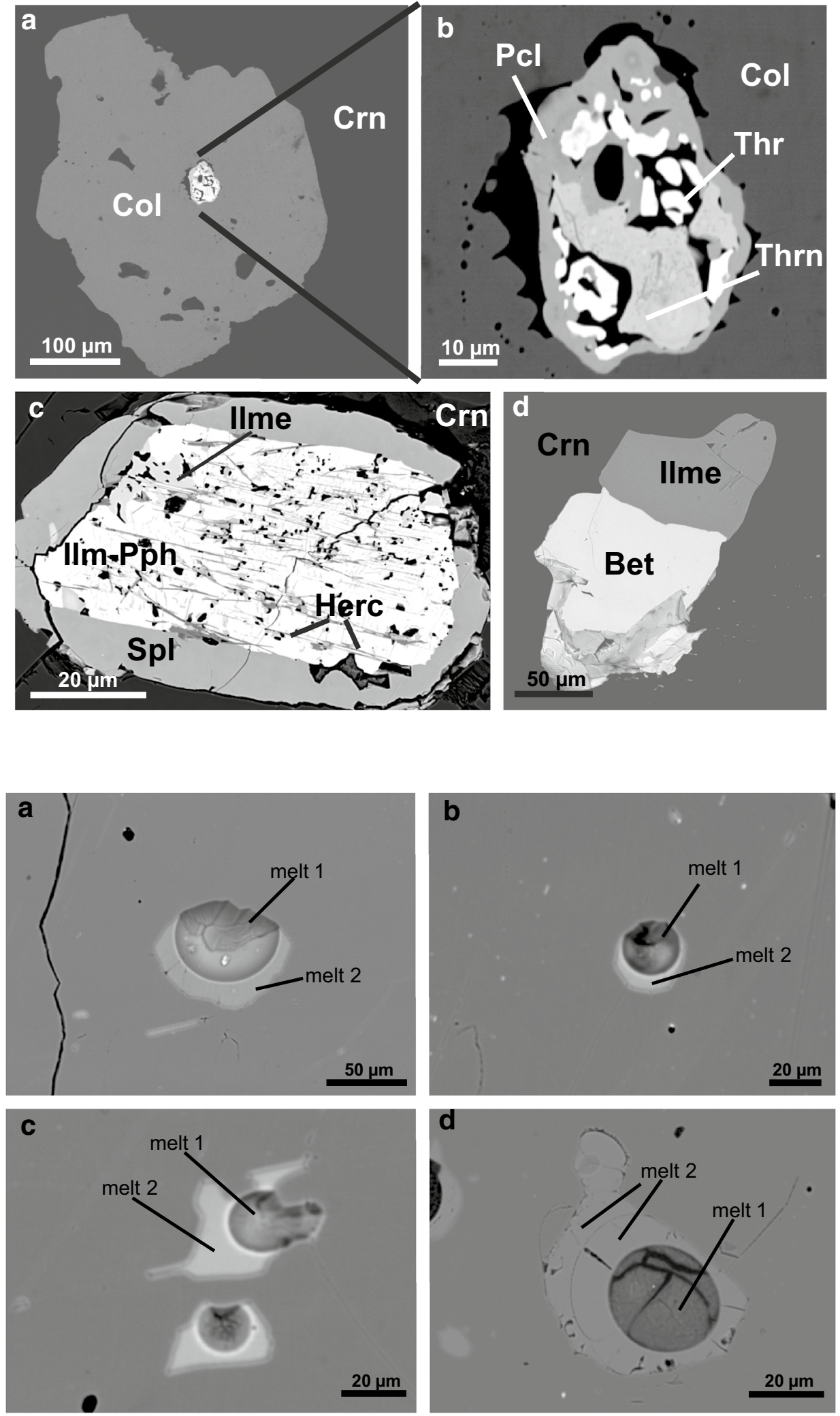

Fig. 6 Inclusions of glasses that represent quenched products of two immiscible melts. Melt 1 which has the composition of an inhomogeneous carbonated silicate melt, forms spherical droplets in melt 2 which has a high-alumina phonolitic composition. Halos around melt 2 are fluorescence effects
Palke et al. 2016, 2017) report melt inclusions (MI) that are $\mathrm{SiO}_{2}$ saturated silicates ranging from trachyandesitic to rhyolitic in composition and, are, hence different than those from the Siebengebirge sapphires. Similar compositions of MI were reported by Song and $\mathrm{Hu}$ (2009) from basalt-hosted sapphires from Changle, China, which are 
Table 4 Results of EPMA analyses of the immiscible melt inclusions in sample 26. Values for $\mathrm{CO}_{2}$ are calculated from the difference to the totals of the EPMA analyses

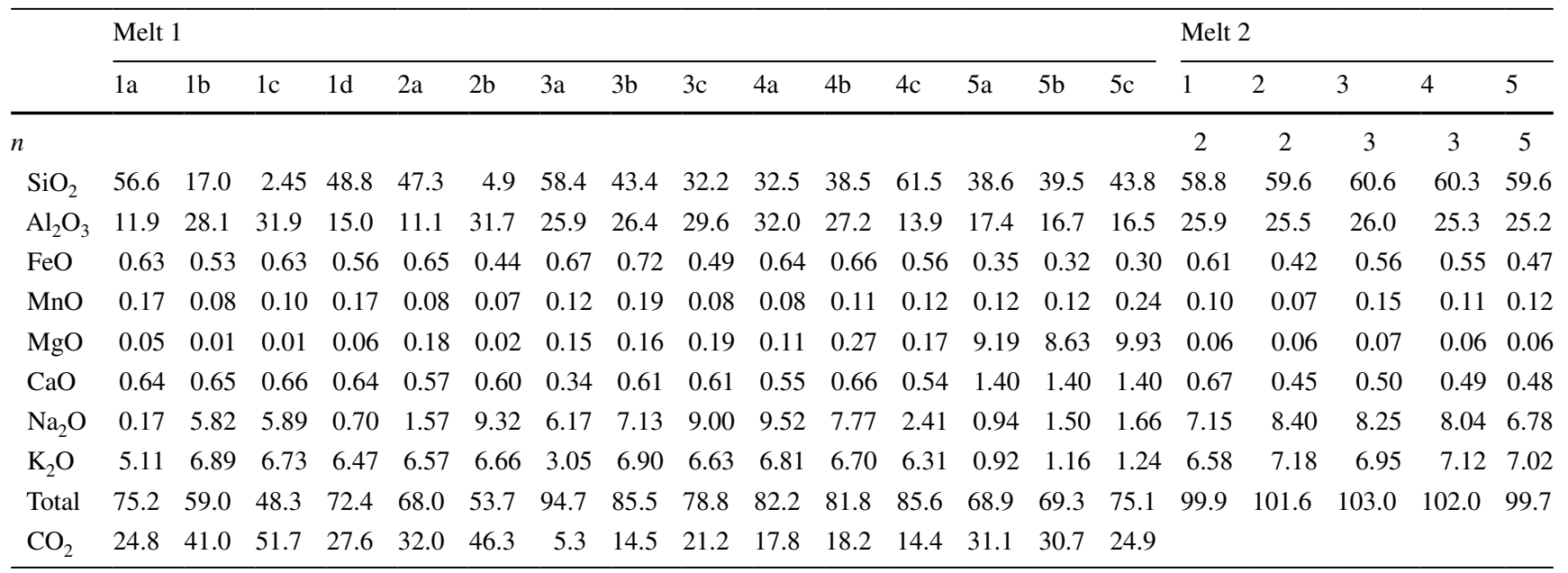

rich in carbonates and sulfates, and by Vysotskij and Barkar (2009) who found primary MI of phonolitic composition in alluvial corundum from the Primorje Region, Russia.

\section{Fluid inclusions}

Fluid inclusions were found in samples 15, 26, S52, and KS21. They occur either randomly distributed within the whole sapphire without any preferred crystallographic orientation, or arranged along trails (Fig. 7a). Sizes of the fluid inclusions are highly variable and range from $<1 \mu \mathrm{m}$ to several tens of micrometers. Many fluid inclusions show negative crystal shapes (Fig. 7b) following the crystal symmetry of the host sapphire, indicating them to be of primary origin.

Many fluid inclusions seem to be pure $\mathrm{CO}_{2}$ with two strong vibrational bands at 1285 and at $1388 \mathrm{~cm}^{-1}$ (Rosso and Bodnar 1995). Some fluid inclusions contain daughter phases (Fig. 7c, d) whose compositions are hard to characterize with Raman spectroscopy due to the overlap between the vibrational spectra of multiple phases at low wavenumbers as well as with the spectrum of the host sapphire. One strong band at $992.4 \mathrm{~cm}^{-1}$ repeatedly occurs in the vibrational spectrum of many fluid inclusions. This band is characteristic for minerals such as natroalunite $\mathrm{NaAl}_{3}\left(\mathrm{SO}_{4}\right)_{2}\left(\mathrm{OH}_{6}\right)$, thenardite $\mathrm{Na}_{2}\left(\mathrm{SO}_{4}\right)$, aphthitalite $\mathrm{K}_{3} \mathrm{Na}\left(\mathrm{SO}_{4}\right)_{2}$, and blödite $\mathrm{Na}_{2} \mathrm{Mg}\left(\mathrm{SO}_{4}\right)_{2} * 4 \mathrm{H}_{2} \mathrm{O}$ (Fig. 8). Further identified daughter minerals are burkeite $\mathrm{Na}_{4}\left(\mathrm{SO}_{4}\right)\left(\mathrm{CO}_{3}\right)$, paravauxite $\mathrm{Fe}^{2+} \mathrm{Al}_{2}\left(\mathrm{PO}_{4}\right)_{2}(\mathrm{OH})_{2} * 8 \mathrm{H}_{2} \mathrm{O}$, and dawsonite $\mathrm{NaAl}\left(\mathrm{CO}_{3}\right)$ $(\mathrm{OH})_{2}$. Although an unambiguous identification of the daughter minerals is not possible from the vibrational spectrum, it can be stated with some confidence that the fluid trapped in the inclusions was a complex sulfatephosphate- $\mathrm{H}_{2} \mathrm{O}$ bearing $\mathrm{CO}_{2}$ fluid with some dissolved cations, notably alumina and alkalis. Similar melt inclusions daughter phases including sulfates and carbonates were reported by Song and $\mathrm{Hu}$ (2009) from the Changle sapphires.

\section{Nano-inclusions}

Selected TEM images of the brown (Nb-Ta-enriched) and blue sapphire domains of sample PE25 are shown in Fig. 9. The brown sapphire domains exhibit a very high population density of nano-inclusions that are probably responsible for the brown coloration. The inclusions form $\sim 10$ to $20 \mathrm{~nm}$ long rods with a shape-preferred orientation having the long axes of the needles near parallel to corundum [0001], though the scatter of the orientation of the long axes (Fig. 9 a, b; TEM bright-field and dark-field images) demonstrates that the nano-inclusions do not show a strict orientation relation relative to the crystal lattice of the host sapphire and thus must be discrete phases. The crystallinity of the nano-inclusions is verified by electron diffraction and dark-field imaging using a reflection from the inclusions (Fig. 9b; TEM darkfield image), lattice fringes from the inclusions and by the Moirée interference patterns visible in the high-resolution image (HRTEM) in Fig. 9c. The latter are produced by the superposition of the crystal lattices of the nano-inclusions and that of the host sapphire. The observed diffraction contrast around the inclusions is characteristic of the so-called Guinier-Preston Zones which are nano-crystalline platelets in a host material. The compositions of the inclusions were qualitatively estimated by energy dispersive methods. All analyzed inclusions are high in $\mathrm{Nb}, \mathrm{Ta}, \mathrm{Ti}$, and $\mathrm{Fe}$. Given that $\mathrm{Nb}$-rich rutile $(\mathrm{Nb}, \mathrm{Ti}, \mathrm{Fe}) \mathrm{O}_{2}$ is a common macroscopic 
Fig. 7 Fluid inclusions in Siebengebirge sapphires. a Crystallographically oriented elongated fluid inclusions. b Trails of fluid inclusions. c, d Multiple phase fluid inclusions
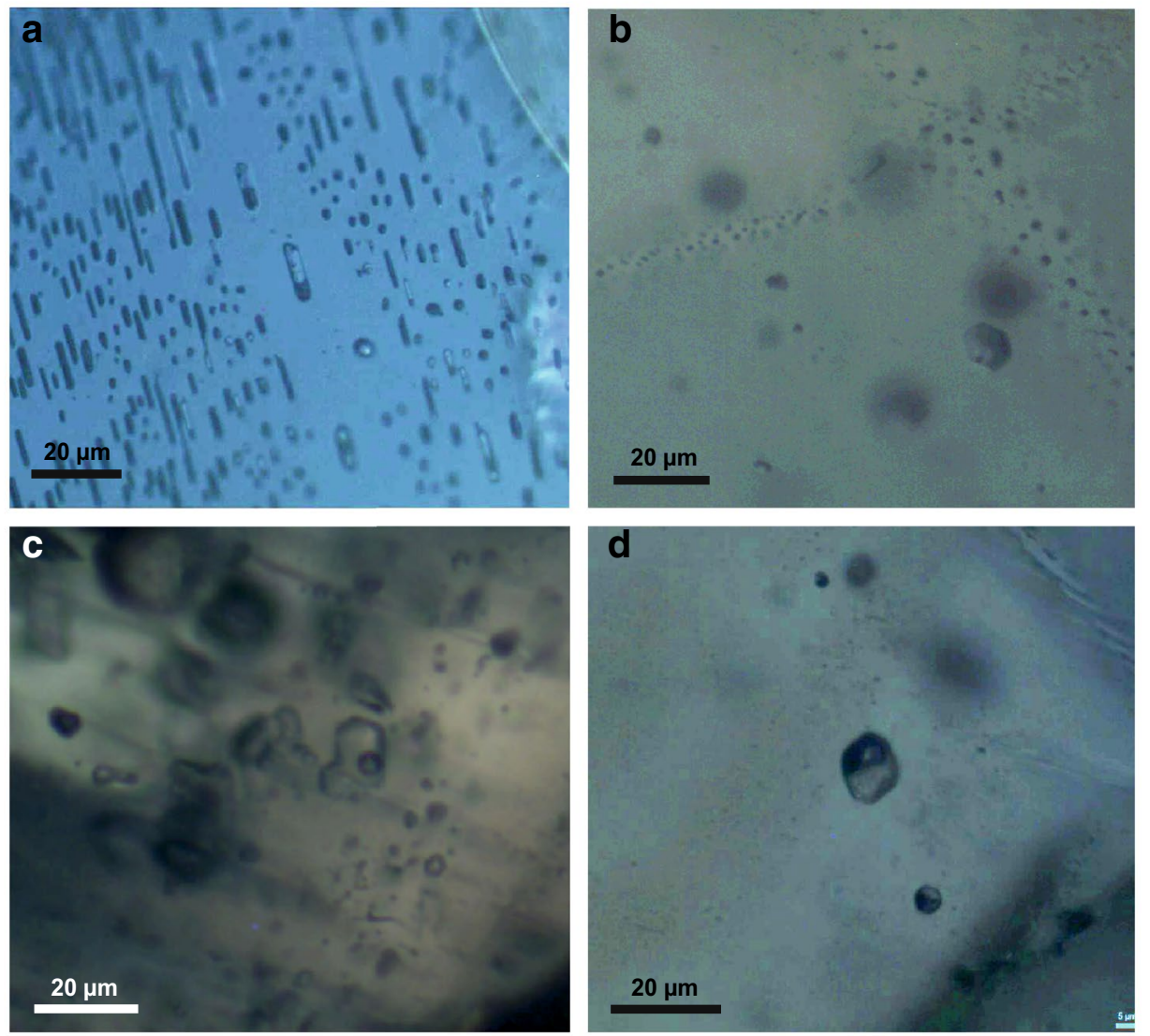

mineral inclusion in the Siebengebirge sapphires, we consider it most likely that Ta-bearing Nb-rich rutile is the phase forming the majority of the nano-inclusions.

The nano-inclusions may be either solid-state exsolution products or they may be primary crystals that grew epitaxially along with the sapphire. The lack of a preferred crystallographic orientation relation between the nanoinclusions and the host sapphire lattice supports the latter possibility, as it has also been argued for platinum group mineral nano-inclusions (nPGM) in pentlandite from the Merensky Reef platinum group element ore deposit of the Bushveld Complex in South Africa by Wirth et al. (2013) and Junge et al. (2014). An additional argument opposing the nano-inclusions to be solid-state exsolutions is provided by their large-scale distribution within the sapphire. Figure 9d (TEM bright-field image) shows that the population density of inclusions varies periodically with inclusion-rich zones intermittently alternating with inclusion-poor zones. Strikingly, the boundaries between the zones with different population densities are very sharp and almost perfectly parallel to each other. The orientation of the lamellar zone/ boundaries is near parallel to [0001] of the corundum lattice and hence may be parallel to the (11-20) hexagonal prism of corundum. The distribution of the nano-inclusions within the sapphire differs significantly from the distribution of larger rutile inclusions that have a needlelike shape and show a strong crystallographic orientation relationship with the host sapphire (Fig. 9e, f).

\section{Geochronology}

The Columbite Group mineral dated in this study is a 200-500 $\mu \mathrm{m}$ sized crystal with the formula $\mathrm{Fe}_{0.2} \mathrm{Mn}_{0.5} \mathrm{Nb}_{2.3} \mathrm{O}_{6}$, suggesting columbite-Mn and was found as an inclusion in sapphire 26 from the Oelberg. As it contained a complex oxide aggregate (Fig. 5a, b), it was repolished until it was free of inclusions which allowed for in situ LA-ICP-MS dating. The reliability of in situ U-Pb dating with LA-ICP-MS has previously been demonstrated by Smith et al. (2004), Dill et al. (2007), Melcher et al. (2008, 2015), and Che et al. (2015) who dated coltan (columbite-tantalite solid solution series) from different pegmatites in China using LA-ICP-MS.

The returned weighted mean ages of the columbite-Mn inclusion (Table 5) are $24.74 \pm 0.24 \mathrm{Ma}$ $(\mathrm{MSWD}=3.4)$ (Fig. 10). The accuracy and reproducibility were checked by repeated analyses of Coltan 007 $(n=8)$ which yields an intercept age of $2074.1 \pm 7.5 \mathrm{Ma}$ 


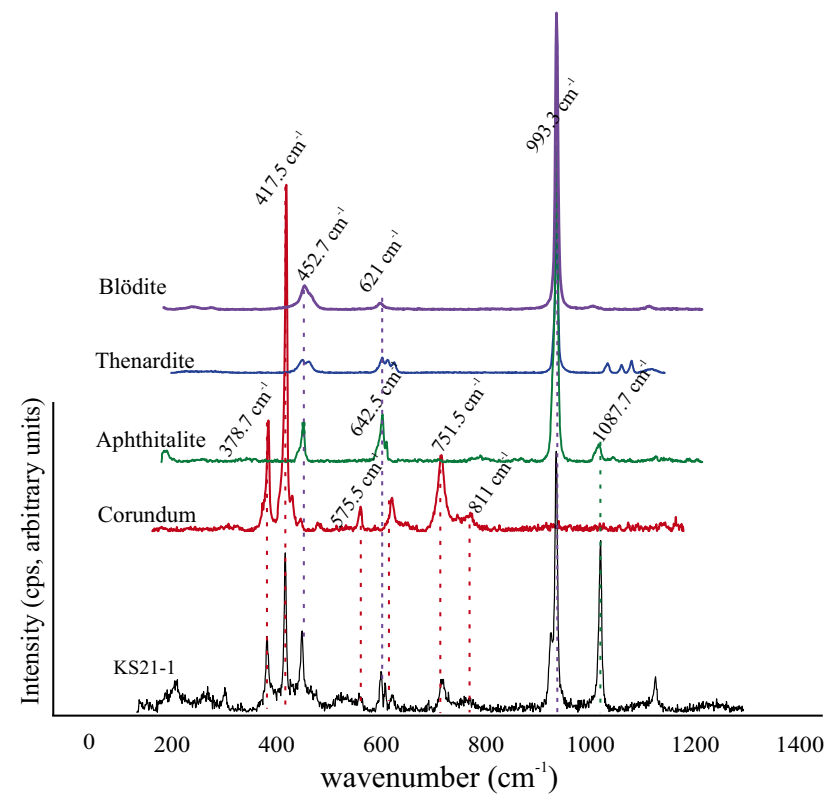

Fig. 8 Raman spectrum of fluid inclusion KS21-1 with the reference spectra of possible daughter phases. The raman spectrum of KS21-1 is a mixed spectrum of several minerals. Many bands represent the corundum matrix. Other bands cannot be unambiguously assigned to a certain mineral. But certainly, the daughter phase must be a sodium sulfate. The reference spectra are from the RRUFF Database. The following reference spectra were used: blödite: R050341; thenardite: R040178; aphthitalite: R050651; corundum: R060020

$(\mathrm{MSWD}=2.9)$ that is in excellent agreement with the published value (Melcher et al. 2008, 2015).

\section{Discussion}

All sapphires investigated in this study are rimmed by tens to several hundred micrometer sized spinel rims, indicating chemical disequilibrium with the host basalt and supporting a xenocrystic nature of the sapphires. This is supported by the phase relations in the CMAS system (Sen and Presnall 1984; Milholland and Presnall 1998; Liu and Presnall 1990, 2000), showing that a hypothetical cooling path of a basaltic model melt will never intersect the corundum stability field (Fig. 11). In the presence of $\mathrm{MgO}$ (in $\mathrm{CMAS}$ ) or $\mathrm{MgO}+\mathrm{FeO}$ (in basalt), stable aluminous phases co-existing with near-liquidus melts are spinel at 1-2 GPa, garnet at $\sim 3 \mathrm{GPa}$, or anorthite at pressures $<1 \mathrm{GPa}$. Hence, the stability of corundum is not only a function of the alumina content of a melt, but is also sensitive to the presence of $\mathrm{MgO}$ and $\mathrm{FeO}$. In a primitive basaltic melt with high $\mathrm{MgO}$ and $\mathrm{FeO}$ contents, the corundum stability field in the CMAS system will never be intersected upon cooling from magmatic temperatures, so that corundum will never be a stable alumina phase.
Questions addressed in the discussion of this paper are the nature of the parental melt that crystallized the sapphires, as well as the relationship between the sapphires and the host primitive alkaline basalts, as sapphires only occur in such primitive basalts from intra-continental settings, but never in basalts from other geotectonic settings.

\section{Magmatic vs. metamorphic origin}

Accepting a xenogenetic relation between the sapphires and the host basalt, the first question to be answered is the geological nature of the Siebengebirge sapphires, i.e., if they formed via igneous or metamorphic processes. The distinction between sapphire crystals of magmatic and of metamorphic origin is mainly based on their $\mathrm{Ga}$ / $\mathrm{Mg}$ ratio (Peucat et al. 2007), but also on the mineral inclusion suite (e.g., Sutherland and Schwarz 2001; Graham et al. 2004). According to Peucat et al. (2007), magmatic blue sapphires have rather high $\mathrm{Ga} / \mathrm{Mg}$ ratios $(>10)$ and metamorphic or metasomatic blue sapphires have rather low $\mathrm{Ga} / \mathrm{Mg}$ ratios $(<10)$. According to that discrimination, the Siebengebirge sapphires would mostly fall into the "magmatic" field, whereas three samples (26, KS21, OL60) would clearly plot in the "metamorphic" field. This discrimination between magmatic and metamorphic sapphires by Peucat et al. (2007) is based on the fractionation of $\mathrm{Ga} / \mathrm{Al}$ and of $\mathrm{Mg}$ in sapphires during crystallization. In the corundum crystal lattice, Ga substitutes for $\mathrm{Al}$, whereas $\mathrm{Mg}$ is located at interstitial lattice sites. Wahlen et al. (1987) found that magmatic corundum generally has higher $\mathrm{Ga} / \mathrm{Al}$ ratios than metamorphic corundum, explaining this by the extraction of $\mathrm{Ga}$ from the metamorphic rocks by circulating F-rich fluids during partial melting of granulites. During fluid circulation, $\mathrm{F}^{-}$would complex with $\mathrm{Ga}^{3+}$ to form $\mathrm{GaF}_{6}^{3-}$ ions that are extracted, leaving the restitic granulite depleted in Ga over Al. If this process implicated by Whalen et al. (1987) controlled the $\mathrm{Ga} / \mathrm{Mg}$ ratio of a sapphire and hence indicated a metamorphic or magmatic origin, the $\mathrm{Ga} / \mathrm{Mg}$ ratio should be a function of the Ga content of the sapphire. Such a Ga control of the $\mathrm{Ga} / \mathrm{Mg}$ ratio cannot be observed for the Siebengebirge sapphires. In contrast, the $\mathrm{Ga} / \mathrm{Mg}$ ratio is correlated with the bulk $\mathrm{Mg}$ content of the sapphire (Fig. 12). Therefore, we consider it unlikely that the Siebengebirge sapphires represent a polymodal suite, i.e., that some samples were formed via metamorphic processes and others are igneous precipitates.

Support for a monogenetic, magmatic, origin for all Siebengebirge sapphires is provided by several other mineralogical characteristics which are (1) syngenetic mineral inclusions of Pyrochlore Group, Columbite Group, calcite and feldspar series members that are more typical for a magmatic origin, (2) the presence of glassy inclusions 

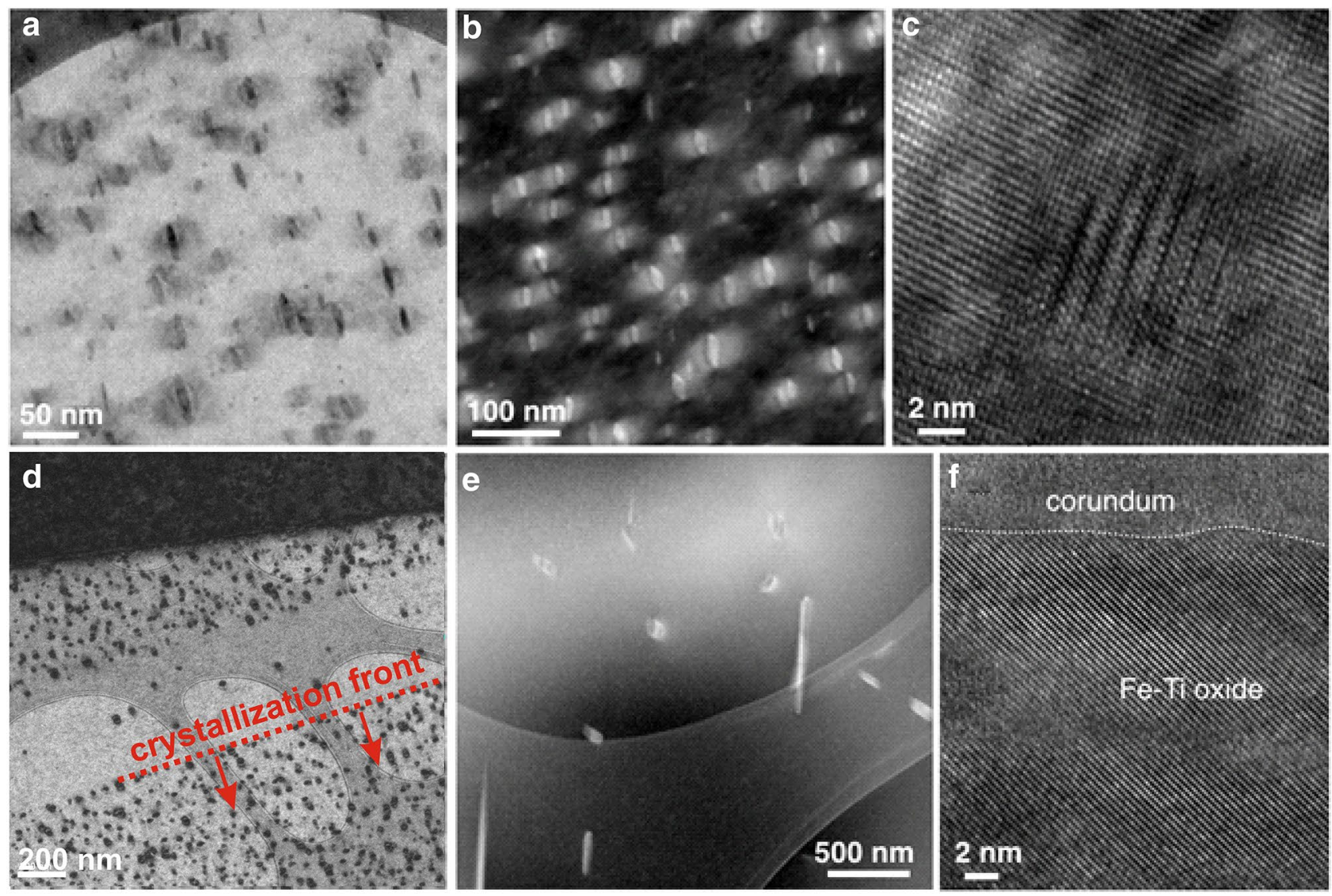

Fig. 9 TEM images of the brownish sapphire domains (a-d) and of the blue sapphire domain (e, f). a TEM bright-field image, and b TEM dark-field image of a zone with a high population density in nano-inclusions, showing that the inclusions have roughly the same shape orientation, but are not in a strict shape-preferred orientation relationship with the host sapphire. Halos around the inclusions are nano-crystalline platelets in the host rock. c high-resolution (HR) TEM lattice fringe image of a nano-inclusion showing typical Moirée

which were likely melts that were trapped as the corundum crystals grew, and (3) the presence of numerous $\mathrm{Nb}$-rich nano-inclusions in the brownish domain of sapphire PE25 which are most likely magmatic precipitates that co-precipitated with the sapphire host during crystal growth. This lamellar distribution of intermittently alternating inclusionpoor and inclusion-rich zones of the nano-inclusions parallel to the prism faces of the host sapphire supports an epitaxial growth of the inclusions on the sapphire crystal faces. Such oscillatory patterns are ubiquitous in many geochemical systems (L'Heureux 2013) and are either interpreted to be externally controlled and to reflect periodic chemical changes in the crystallizing environment, or to be internally controlled by intrinsic self-organization in a nonequilibrium system with positive feedback (e.g., Ortoleva 1994; Merino and Wang 2001).

Perhaps the most compelling evidence for a magmatic origin for the Siebengebirge sapphires comes from the age patterns. d TEM bright-field image showing a low-population density zone between two zones of high nano-inclusion population density. The zone-boundary is sharp and oriented parallel to the [0001] zone axis of the host sapphire. e TEM bright-field image of a blue sapphire domain showing strictly crystallographically oriented needle-like ilmenite inclusions. f HRTEM image of a blue domain showing the lattice fringe of an ilmenite inclusion within corundum

of their inclusions. If the sapphires and hence their mineral inclusions had been of metamorphic origin, then they should yield Devonian ages (i.e., the age of the metamorphic basement below the SVF). However, our own in situ dating resulted in Cenozoic ages which, when taking into account the uncertainty, are the same age as the host basalt $(24.74 \pm 0.24 \mathrm{Ma})$, indicating a clear magmatic origin for these inclusions and hence for their host sapphires.

Based on this discussion, we state that the discrimination between magmatic and metamorphic sapphires based on the $\mathrm{Ga} / \mathrm{Mg}$ ratio as proposed by Peucat et al. (2007) has to be regarded with caution and may not be applied to any sapphire without additional information provided by the mineral inclusion suite. The $\mathrm{Ga} / \mathrm{Mg}$ ratio may be used as a discrimination feature for the sapphire samples investigated in the study from Peucat et al. (2007), but at least for the sapphires presented in this study this may not be applicable. Similar discrepancies for the discrimination between "magmatic" 
Table $5 \mathrm{U}-\mathrm{Th}-\mathrm{Pb}$ isotopic data for the columbite-Mn inclusion

\begin{tabular}{|c|c|c|c|c|c|c|c|c|c|c|c|c|c|c|c|c|}
\hline \multirow[t]{3}{*}{ Spot } & \multirow{3}{*}{$\begin{array}{l}{ }^{207} \mathrm{~Pb}^{\mathrm{a}} \\
(\mathrm{cps})\end{array}$} & \multirow{3}{*}{$\begin{array}{l}\mathrm{U}^{\mathrm{b}} \\
(\mathrm{ppm})\end{array}$} & \multirow{3}{*}{$\begin{array}{l}\mathrm{Pb}^{\mathrm{b}} \\
(\mathrm{ppm})\end{array}$} & \multirow{3}{*}{$\begin{array}{l}\underline{T h}^{\underline{b}} \\
U\end{array}$} & \multirow{3}{*}{$\begin{array}{l}{ }^{206} \mathrm{Pbc}^{\mathrm{c}} \\
(\%)\end{array}$} & \multirow{3}{*}{$\begin{array}{l}{ }^{206} \underline{\mathrm{Pb}}^{\underline{\mathrm{d}}} \\
{ }^{238} \mathrm{U}\end{array}$} & \multirow{3}{*}{$\begin{array}{l} \pm 2 \sigma \\
(\%)\end{array}$} & \multirow{3}{*}{$\begin{array}{l}{ }^{207} \underline{\mathrm{Pb}}^{\mathrm{d}} \\
{ }^{235} \mathrm{U}\end{array}$} & \multirow{3}{*}{$\begin{array}{l} \pm 2 \sigma \\
(\%)\end{array}$} & \multirow{3}{*}{$\begin{array}{l}{ }^{207} \underline{\mathrm{Pb}}^{\mathrm{d}} \\
{ }^{206} \mathrm{~Pb}\end{array}$} & \multirow{3}{*}{$\begin{array}{l} \pm 2 \sigma \\
(\%)\end{array}$} & \multirow{3}{*}{$\mathrm{rho}^{\mathrm{e}}$} & \multirow{3}{*}{$\begin{array}{l}\text { Age } \\
{ }^{206} \underline{\mathrm{Pb}} \\
{ }^{238} \mathrm{U}\end{array}$} & \multirow{3}{*}{$\begin{array}{l} \pm 2 \sigma \\
(\mathrm{Ma})\end{array}$} & \multirow{3}{*}{$\begin{array}{l}\text { Age } \\
{ }^{207} \underline{\mathrm{Pb}} \\
{ }^{235} \mathrm{U}\end{array}$} & \multirow{3}{*}{$\begin{array}{l} \pm 2 \sigma \\
\text { (Ma) }\end{array}$} \\
\hline & & & & & & & & & & & & & & & & \\
\hline & & & & & & & & & & & & & & & & \\
\hline A06 & 569 & 685 & 2.9 & 0.66 & 1.4 & 0.00368 & 3.3 & 0.0229 & 9.5 & 0.0451 & 8.9 & 0.35 & 23.7 & 0.8 & 23.0 & 2.2 \\
\hline A07 & 367 & 363 & 1.5 & 0.47 & 1.8 & 0.00383 & 3.8 & 0.0248 & 8.0 & 0.0470 & 7.1 & 0.47 & 24.7 & 0.9 & 24.9 & 2.0 \\
\hline A08 & 513 & 687 & 3.0 & 0.61 & 0.9 & 0.00397 & 3.5 & 0.0253 & 9.1 & 0.0462 & 8.3 & 0.39 & 25.5 & 0.9 & 25.3 & 2.3 \\
\hline A09 & 402 & 419 & 1.8 & 0.44 & 2.1 & 0.00397 & 3.7 & 0.0250 & 9.2 & 0.0457 & 8.5 & 0.40 & 25.6 & 0.9 & 25.1 & 2.3 \\
\hline A10 & 26,135 & 384 & 20.7 & 0.58 & 25.9 & 0.00385 & 11 & 0.0270 & 17.3 & 0.0509 & 13 & 0.63 & 24.8 & 2.7 & 27.1 & 4.6 \\
\hline A11 & 375 & 249 & 1.1 & 0.34 & 4.0 & 0.00394 & 4.3 & 0.0248 & 12.0 & 0.0456 & 11 & 0.35 & 25.4 & 1.1 & 24.9 & 3.0 \\
\hline $\mathrm{A} 12$ & 1230 & 616 & 3.5 & 0.85 & 10.1 & 0.00393 & 3.9 & 0.0255 & 12.1 & 0.0471 & 11 & 0.32 & 25.3 & 1.0 & 25.5 & 3.1 \\
\hline A13 & 813 & 570 & 2.7 & 0.50 & 7.0 & 0.00383 & 3.8 & 0.0232 & 13.8 & 0.0440 & 13 & 0.27 & 24.6 & 0.9 & 23.3 & 3.2 \\
\hline A14 & 727 & 623 & 3.0 & 0.69 & 3.9 & 0.00387 & 3.3 & 0.0240 & 8.6 & 0.0450 & 8 & 0.38 & 24.9 & 0.8 & 24.1 & 2.0 \\
\hline A15 & 849 & 946 & 4.8 & 1.02 & 0.3 & 0.00401 & 3.2 & 0.0266 & 5.8 & 0.0481 & 4.8 & 0.56 & 25.8 & 0.8 & 26.7 & 1.5 \\
\hline A16 & 7954 & 544 & 7.9 & 0.68 & 25.0 & 0.00378 & 5.4 & 0.0243 & 18.6 & 0.0467 & 18 & 0.29 & 24.3 & 1.3 & 24.4 & 4.5 \\
\hline A17 & 282 & 269 & 1.1 & 0.28 & 5.1 & 0.00383 & 4.3 & 0.0221 & 16.6 & 0.0419 & 16 & 0.26 & 24.7 & 1.1 & 22.2 & 3.6 \\
\hline A18 & 373 & 365 & 1.5 & 0.34 & 1.4 & 0.00375 & 3.9 & 0.0245 & 8.9 & 0.0473 & 8.1 & 0.43 & 24.2 & 0.9 & 24.5 & 2.2 \\
\hline A19 & 507 & 230 & 0.9 & 0.25 & 3.0 & 0.00388 & 3.3 & 0.0238 & 11.7 & 0.0445 & 11 & 0.28 & 25.0 & 0.8 & 23.9 & 2.8 \\
\hline $\mathrm{A} 20$ & 865 & 402 & 1.6 & 0.49 & 3.3 & 0.00368 & 3.2 & 0.0256 & 9.8 & 0.0504 & 9.3 & 0.33 & 23.7 & 0.8 & 25.6 & 2.5 \\
\hline $\mathrm{A} 21$ & 1198 & 492 & 2.0 & 0.29 & 4.4 & 0.00376 & 3.2 & 0.0251 & 7.3 & 0.0484 & 6.5 & 0.44 & 24.2 & 0.8 & 25.2 & 1.8 \\
\hline A22 & 1251 & 422 & 1.9 & 0.34 & 6.2 & 0.00400 & 3.2 & 0.0264 & 7.4 & 0.0480 & 6.6 & 0.44 & 25.7 & 0.8 & 26.5 & 1.9 \\
\hline A23 & 1242 & 371 & 1.7 & 0.56 & 7.2 & 0.00370 & 3.7 & 0.0234 & 11.2 & 0.0459 & 11 & 0.33 & 23.8 & 0.9 & 23.5 & 2.6 \\
\hline A24 & 694 & 325 & 1.4 & 0.51 & 2.4 & 0.00377 & 3.2 & 0.0241 & 8.9 & 0.0463 & 8.3 & 0.35 & 24.3 & 0.8 & 24.2 & 2.1 \\
\hline A 25 & 771 & 387 & 1.6 & 0.43 & 1.7 & 0.00372 & 3.6 & 0.0247 & 8.3 & 0.0482 & 7.4 & 0.43 & 23.9 & 0.9 & 24.8 & 2.0 \\
\hline A26 & 719 & 406 & 1.6 & 0.44 & 1.7 & 0.00365 & 3.4 & 0.0226 & 8.6 & 0.0450 & 7.9 & 0.40 & 23.5 & 0.8 & 22.7 & 1.9 \\
\hline $\mathrm{A} 27$ & 444 & 457 & 2.1 & 0.57 & 1.1 & 0.00406 & 3.4 & 0.0263 & 9.5 & 0.0471 & 8.9 & 0.36 & 26.1 & 0.9 & 26.4 & 2.5 \\
\hline A 28 & 422 & 451 & 1.9 & 0.44 & 2.4 & 0.00390 & 3.6 & 0.0238 & 9.7 & 0.0442 & 9 & 0.37 & 25.1 & 0.9 & 23.9 & 2.3 \\
\hline A29 & 417 & 428 & 1.8 & 0.57 & 1.2 & 0.00375 & 3.7 & 0.0242 & 9.4 & 0.0469 & 8.6 & 0.39 & 24.1 & 0.9 & 24.3 & 2.3 \\
\hline $\mathrm{A} 30$ & 448 & 505 & 2.2 & 0.52 & 0.9 & 0.00383 & 3.7 & 0.0248 & 9.2 & 0.0470 & 8.5 & 0.40 & 24.6 & 0.9 & 24.9 & 2.3 \\
\hline A31 & 591 & 566 & 2.4 & 0.52 & 1.7 & 0.00375 & 3.4 & 0.0236 & 7.3 & 0.0456 & 6.4 & 0.47 & 24.1 & 0.8 & 23.6 & 1.7 \\
\hline A 32 & 567 & 491 & 2.2 & 0.47 & 4.3 & 0.00395 & 3.2 & 0.0257 & 12.8 & 0.0471 & 12 & 0.25 & 25.4 & 0.8 & 25.7 & 3.3 \\
\hline A38 & 483 & 441 & 2.0 & 0.62 & 2.1 & 0.00378 & 3.7 & 0.0236 & 12.3 & 0.0453 & 12 & 0.30 & 24.3 & 0.9 & 23.7 & 2.9 \\
\hline A39 & 995 & 666 & 2.8 & 0.74 & 3.0 & 0.00374 & 3.4 & 0.0233 & 9.9 & 0.0451 & 9.3 & 0.34 & 24.1 & 0.8 & 23.4 & 2.3 \\
\hline A 40 & 441 & 208 & 0.9 & 0.48 & 1.9 & 0.00402 & 3.9 & 0.0257 & 10.0 & 0.0464 & 9.2 & 0.39 & 25.9 & 1.0 & 25.8 & 2.5 \\
\hline A41 & 473 & 233 & 0.9 & 0.28 & 1.0 & 0.00381 & 3.5 & 0.0264 & 7.8 & 0.0502 & 7 & 0.44 & 24.5 & 0.8 & 26.4 & 2.0 \\
\hline A42 & 725 & 177 & 0.9 & 0.46 & 14.4 & 0.00389 & 3.5 & 0.0258 & 15.4 & 0.0480 & 15 & 0.23 & 25.0 & 0.9 & 25.8 & 3.9 \\
\hline A43 & 556 & 273 & 1.1 & 0.42 & 1.9 & 0.00364 & 3.2 & 0.0236 & 10.8 & 0.0470 & 10 & 0.30 & 23.5 & 0.8 & 23.7 & 2.5 \\
\hline A44 & 769 & 368 & 1.6 & 0.51 & 1.8 & 0.00399 & 3.0 & 0.0259 & 7.9 & 0.0471 & 7.3 & 0.39 & 25.6 & 0.8 & 26.0 & 2.0 \\
\hline A45 & 1158 & 338 & 1.5 & 0.45 & 10.2 & 0.00364 & 3.7 & 0.0226 & 9.9 & 0.0451 & 9.2 & 0.38 & 23.4 & 0.9 & 22.7 & 2.2 \\
\hline A46 & 866 & 379 & 1.7 & 0.47 & 4.4 & 0.00392 & 3.4 & 0.0258 & 9.1 & 0.0478 & 8.4 & 0.38 & 25.2 & 0.9 & 25.9 & 2.3 \\
\hline A47 & 1007 & 400 & 1.8 & 0.44 & 5.4 & 0.00393 & 3.2 & 0.0241 & 8.8 & 0.0445 & 8.2 & 0.36 & 25.2 & 0.8 & 24.2 & 2.1 \\
\hline A48 & 2132 & 478 & 2.5 & 0.45 & 12.6 & 0.00399 & 3.2 & 0.0252 & 9.7 & 0.0458 & 9.1 & 0.34 & 25.7 & 0.8 & 25.3 & 2.4 \\
\hline A49 & 625 & 343 & 1.4 & 0.37 & 1.1 & 0.00382 & 3.2 & 0.0259 & 8.7 & 0.0493 & 8.1 & 0.36 & 24.6 & 0.8 & 26.0 & 2.2 \\
\hline A50 & 942 & 432 & 1.8 & 0.45 & 3.1 & 0.00384 & 3.1 & 0.0252 & 7.7 & 0.0476 & 7.1 & 0.41 & 24.7 & 0.8 & 25.3 & 1.9 \\
\hline A51 & 614 & 276 & 1.1 & 0.32 & 3.3 & 0.00388 & 3.5 & 0.0238 & 10.7 & 0.0445 & 10 & 0.32 & 25.0 & 0.9 & 23.9 & 2.5 \\
\hline
\end{tabular}


Table 5 continued

\begin{tabular}{|c|c|c|c|c|c|c|c|c|c|c|c|c|c|c|c|c|}
\hline \multirow[t]{3}{*}{ Spot } & \multirow{3}{*}{$\begin{array}{l}{ }^{207} \mathrm{~Pb}^{\mathrm{a}} \\
(\mathrm{cps})\end{array}$} & \multirow{3}{*}{$\begin{array}{l}\mathrm{U}^{\mathrm{b}} \\
(\mathrm{ppm})\end{array}$} & \multirow{3}{*}{$\begin{array}{l}\mathrm{Pb}^{\mathrm{b}} \\
(\mathrm{ppm})\end{array}$} & \multirow{3}{*}{$\begin{array}{l}\underline{\mathrm{Th}}^{\underline{\mathrm{b}}} \\
\mathrm{U}\end{array}$} & \multirow{3}{*}{$\begin{array}{l}{ }^{206} \mathrm{Pbc}^{\mathrm{c}} \\
(\%)\end{array}$} & \multirow{3}{*}{$\begin{array}{l}{ }^{206} \underline{\mathrm{Pb}}^{\mathrm{d}} \\
{ }^{238} \mathrm{U}\end{array}$} & \multirow{3}{*}{$\begin{array}{l} \pm 2 \sigma \\
(\%)\end{array}$} & \multirow{3}{*}{$\begin{array}{l}{ }^{207} \underline{\mathrm{Pb}}^{\mathrm{d}} \\
{ }^{235} \mathrm{U}\end{array}$} & \multirow{3}{*}{$\begin{array}{l} \pm 2 \sigma \\
(\%)\end{array}$} & \multirow{3}{*}{$\begin{array}{l}{ }^{207} \underline{\mathrm{Pb}}^{\mathrm{d}} \\
{ }^{206} \mathrm{~Pb}\end{array}$} & \multirow{3}{*}{$\begin{array}{l} \pm 2 \sigma \\
(\%)\end{array}$} & \multirow{3}{*}{$\mathrm{rho}^{\mathrm{e}}$} & \multicolumn{2}{|l|}{ Age } & \multicolumn{2}{|l|}{ Age } \\
\hline & & & & & & & & & & & & & ${ }^{206} \underline{\mathrm{Pb}}$ & $\pm 2 \sigma$ & ${ }^{207} \underline{\mathrm{Pb}}$ & $\pm 2 \sigma$ \\
\hline & & & & & & & & & & & & & ${ }^{238} \mathrm{U}$ & (Ma) & ${ }^{235} \mathrm{U}$ & (Ma) \\
\hline$\overline{\mathrm{A} 52}$ & 693 & 313 & 1.2 & 0.29 & 3.0 & 0.00373 & 3.5 & 0.0255 & 7.6 & 0.0496 & 6.8 & 0.46 & 24.0 & 0.8 & 25.5 & 1.9 \\
\hline A53 & 1023 & 643 & 2.8 & 0.70 & 1.3 & 0.00388 & 3.1 & 0.0266 & 7.3 & 0.0497 & 6.5 & 0.43 & 25.0 & 0.8 & 26.7 & 1.9 \\
\hline A54 & 865 & 345 & 1.5 & 0.33 & 4.0 & 0.00409 & 3.1 & 0.0263 & 10.5 & 0.0467 & 10 & 0.30 & 26.3 & 0.8 & 26.4 & 2.7 \\
\hline A55 & 1920 & 385 & 2.1 & 0.61 & 11.2 & 0.00405 & 3.3 & 0.0292 & 12.4 & 0.0524 & 12 & 0.27 & 26.0 & 0.9 & 29.3 & 3.6 \\
\hline A56 & 41,353 & 1981 & 145.2 & 0.02 & 0.0 & 0.07940 & 2.6 & 0.6281 & 2.8 & 0.0574 & 0.99 & 0.93 & 492.6 & 12.3 & 494.9 & 10.9 \\
\hline
\end{tabular}

Spot size $=19$ and $28 \mu \mathrm{m}$, respectively; depth of crater $\sim 20 \mu \mathrm{m} .{ }^{206} \mathrm{~Pb} /{ }^{238} \mathrm{U}$ error is the quadratic addition of the within run precision ( $2 \mathrm{SE}$ ) and an estimated repeatability of $2 \%(2 \mathrm{SD}) .{ }^{207} \mathrm{~Pb} /{ }^{206} \mathrm{~Pb}$ error propagation $\left({ }^{207} \mathrm{~Pb}\right.$ signal dependent) following Gerdes and $\mathrm{Zeh}(2009) .{ }^{207} \mathrm{~Pb} /{ }^{235} \mathrm{U}$ error is the quadratic addition of the ${ }^{207} \mathrm{~Pb} /{ }^{206} \mathrm{~Pb}$ and ${ }^{206} \mathrm{~Pb} /{ }^{238} \mathrm{U}$ uncertainty

${ }^{a}$ Within run background-corrected mean ${ }^{207} \mathrm{~Pb}$ signal in cps (counts per second)

${ }^{b} \mathrm{U}$ and $\mathrm{Pb}$ content and $\mathrm{Th} / \mathrm{U}$ ratio were calculated relative to $\mathrm{GJ}-1$ reference zircon

c Percentage of the common $\mathrm{Pb}$ on the ${ }^{206} \mathrm{~Pb}$. b.d.l. below detection limit

${ }^{\mathrm{d}}$ Corrected for background, within-run $\mathrm{Pb} / \mathrm{U}$ fractionation (in case of ${ }^{206} \mathrm{~Pb} /{ }^{238} \mathrm{U}$ ) and common $\mathrm{Pb}$ using Stacey and $\mathrm{Kramers}(1975$ ) model $\mathrm{Pb}$ composition and subsequently normalised to GJ-1 (ID-TIMS value/measured value); ${ }^{207} \mathrm{~Pb} /{ }^{235} \mathrm{U}$ calculated $u$ sing ${ }^{207} \mathrm{~Pb} /{ }^{206} \mathrm{~Pb} /$ $\left({ }^{238} \mathrm{U} /{ }^{206} \mathrm{~Pb} * 1 / 137.88\right)$

${ }^{\text {e }}$ Rho is the ${ }^{206} \mathrm{~Pb} /{ }^{238} \mathrm{U} /{ }^{207} \mathrm{~Pb} /{ }^{235} \mathrm{U}$ error correlation coefficient

${ }^{\mathrm{f}}$ Degree of concordance $={ }^{206} \mathrm{~Pb} /{ }^{238} \mathrm{U}$ age $/{ }^{207} \mathrm{~Pb} /{ }^{206} \mathrm{~Pb}$ age $\times 100$

$\mathrm{g}$ Accuracy and reproducibility were checked by repeated analyses ( $n=8$ and 9 ) of reference columbite 139 and 007 ; data given as mean with 2 standard deviation uncertainties

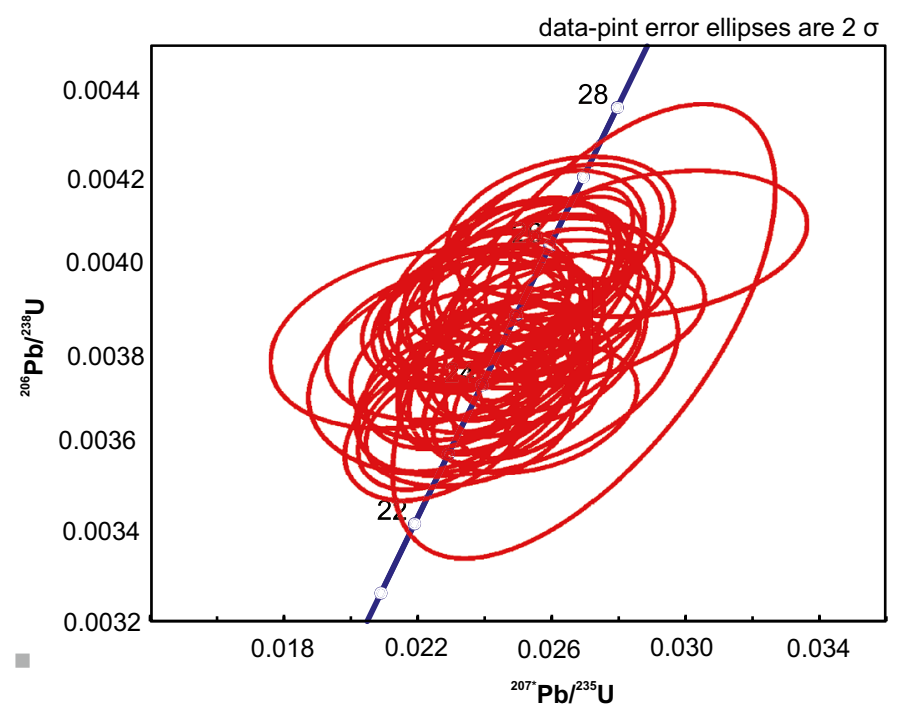

Fig. 10 Age calculations for the columbite-Mn inclusion in the Siebengebirge sapphire sample 26. a Data plotted in a Wetherill concordia diagram. b Weighted mean age based on the calculated ${ }^{206} \mathrm{~Pb} /{ }^{238} \mathrm{U}$

and "metamorphic" sapphires based on their Ga content, and the $\mathrm{Ga} / \mathrm{Mg}$ ratio as proposed by Peucat et al. (2007) were pointed out by Izokh et al. (2010), Uher et al. (2012), Sutherland et al. (2015), and Palke et al. (2015, 2017). The discrepancies in trace element data and the resulting ambiguity about the origin of sapphires from alluvial deposits in

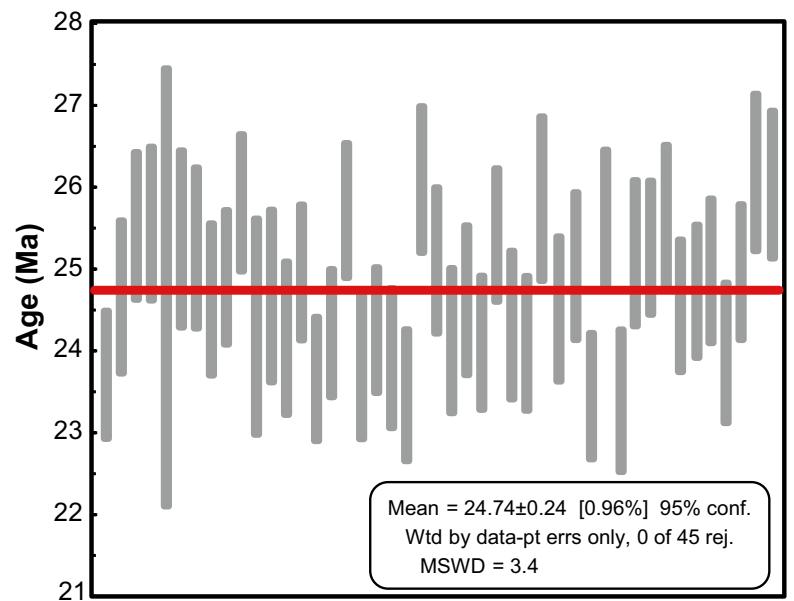

ratio. The gray bars are the errors of the calculated age; the red line represents the mean age of all analyses

Montana, USA, lead Palke et al. (2017) to suggest the trace element classification scheme for sapphires to be revisited and revised. Based on our trace element data on the Siebengebirge sapphires, we support the necessity of the revision of the Peucat et al. (2007) trace element-based discrimination for the identification of the origin of sapphires. 


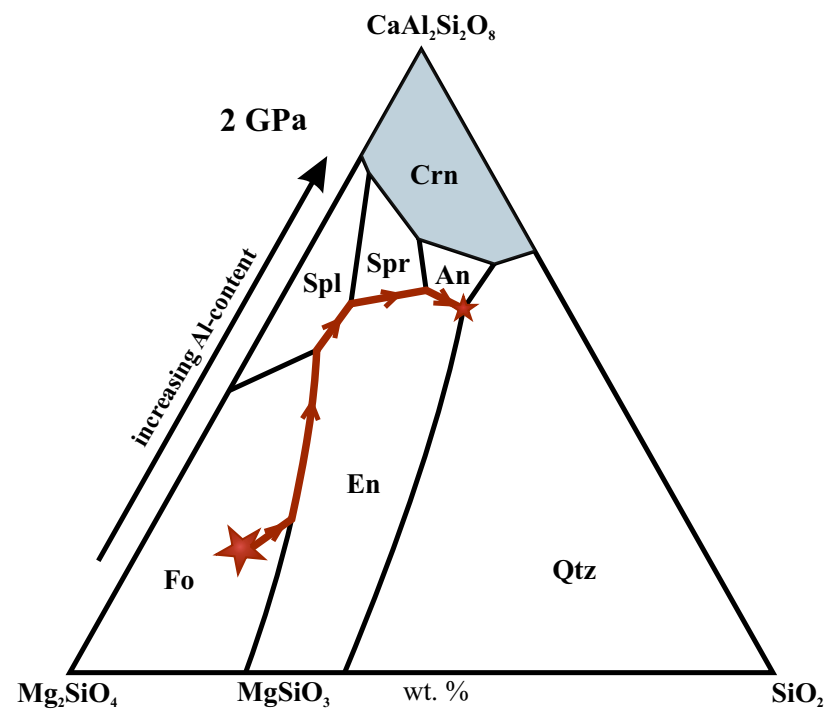

Fig. 11 Phase relations in the CMAS system at 2 GPa (Liu und Presnall 1990) pressures. Abbreviations after Whitney and Evans (2010). The star in the forsterite field represents a typical composition of an alkali basalt. Upon cooling, the melt will follow the red path. The final eutectic composition of the melt is given by the small stars at the anorthite-enstatite-quartz triple point
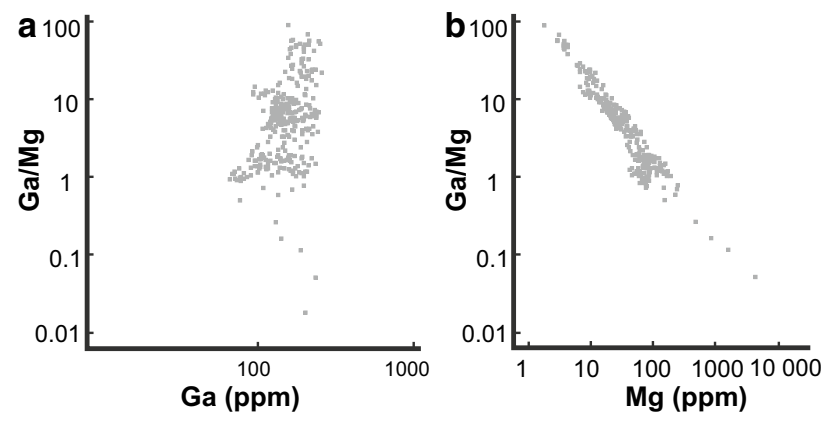

Fig. 12 a $\mathrm{Ga} / \mathrm{Mg}$ vs. $\mathrm{Ga}(\mathrm{ppm})$ and $\mathbf{b} \mathrm{Ga} / \mathrm{Mg}$ vs. $\mathrm{Mg}$ (ppm) plots. There is no visible effect of $\mathrm{Ga}$ on the $\mathrm{Ga} / \mathrm{Mg}$ ratio, whereas the total $\mathrm{Mg}$ content in the sapphires is highly correlated with the $\mathrm{Ga} / \mathrm{Mg}$ ratio, indicating that the strong variability of the $\mathrm{Ga} / \mathrm{Mg}$ ratio in the Siebengebirge sapphires is controlled by changes in the $\mathrm{Mg}$ concentration, and largely unaffected by changes in the Ga content

\section{Constraints on the parental melt}

Important indicators for the composition of the parental melt of the Siebengebirge sapphires are the trace element composition and the composition of syngenetic mineral, fluid, and glass inclusions, as these directly sample the parental melt. One key characteristic of the Siebengebirge sapphires is the abundance of HFSE-rich mineral inclusions and of HFSE-rich nano-inclusions in the brownish domains, indicating that the sapphires' parental melt must have been rich in the HFSE. Such high concentrations in the HFSE are well known from highly evolved silicate melts and from carbonatitic melts. Most authors (e.g., Sutherland et al. 1998b) relate the HFSE enrichment of the sapphires and the presence of Columbite Group, Pyrochlore Group and of $\mathrm{Nb}$-rich rutile mineral inclusions to highly evolved $\mathrm{SiO}_{2}$ undersaturated silicate melts such as syenites, or nepheline syenites. A further observation leading these authors to favor syenites as parental melts is that repeatedly melt inclusions of syenitic composition were reported from magmatic sapphires (e.g., Pakhomova et al. 2006; Zaw et al. 2006; Izokh et al. 2010). Up until now, carbonatites have not been considered as potential parental melts, although many authors (e.g., Coenraads et al. 1990; Van Long et al. 2004; McGee 2005; Nechaev et al. 2009; Izokh et al. 2010; Palke et al. 2017) reported that $\mathrm{CO}_{2}$ must have played a significant role in the formation of these sapphires, as $\mathrm{CO}_{2}$-rich fluid inclusions are commonly observed in many magmatic sapphires. Palke et al. (2017) interpreted the presence of analcime-calcite inclusions in alluvial magmatic sapphires from Montana, USA as an indication for that the sapphires crystallized from a melt that simultaneously exsolved a carbonatite melt, and fractions of that carbonatite melt were trapped by the growing sapphire and later recrystallized to the analcime-calcite paragenesis. However, in their genetic model, the carbonatite melt was only a "by-product" that resulted from the carbonated nature of the source rock, and they did not state a compelling necessity of the presence of $\mathrm{CO}_{2}$ or a carbonatite melt for the sapphire formation. Only Guo et al. (1996) and Limatrakun et al. (2001) assign carbonatitic melts a role in sapphire petrogenesis. Yet, the only role that was assigned to carbonatites in these studies was their function as a $\mathrm{CO}_{2}$ source, but no direct parentage was proposed, mainly due to the lack of primary evidence for the presence of a carbonatitic melt during sapphire crystallization.

The Siebengebirge sapphires by contrast do show direct evidence for the participation of a carbonatitic melt in the sapphire petrogenesis which is given by the several primary carbonate inclusions and by the glasses in sample 26. These glasses have two different compositions and are either a carbonated silicate or a phonolitic glass, and probably represent quenched liquids that formed via liquid immiscibility from a carbonated silicate melt, as indicated by the textures shown in Fig. 6. We, therefore, suggest that liquid immiscibility in the carbonatite-silicate melt system played a major role in the genesis of magmatic sapphires, in a similar fashion as stated before by Palke et al. (2017) for magmatic sapphires from Montana, USA.

The liquid miscibility relations in the carbonatitesilicate system have been studied intensively in the past decades (Koster VanGroos and Wyllie 1963, 1966; Hamilton and Freestone 1979; Freestone and Hamilton 1980; Kjarsgaard and Hamilton 1988; Brooker and Hamilton 


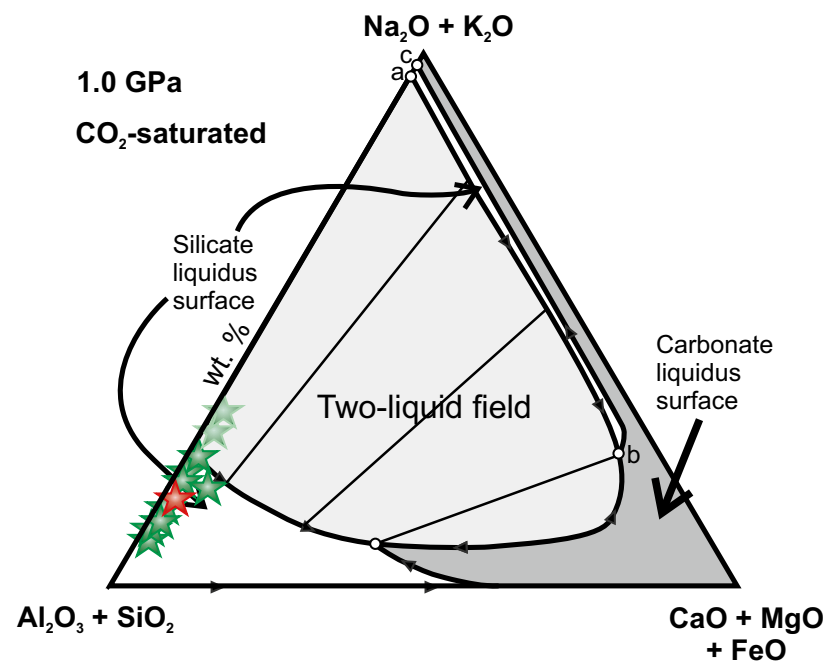

Fig. 13 Location of the glass compositions (melt 1: green stars; melt 2: red star) in the schematic pseudoternary phase diagram of the Hamilton projection at $1 \mathrm{GPa}$ (Lee and Wyllie 1998). The line ' $a-b$ ' represents the two liquid field boundary at the $\mathrm{Na}_{2} \mathrm{O}, \mathrm{K}_{2} \mathrm{O}$ rich side, and line ' $b-c$ ' represents the silicate-carbonate field boundary. The composition of both glasses plot in the same field (silicate liquidus surface at the $\mathrm{SiO}_{2}+\mathrm{Al}_{2} \mathrm{O}_{3}$ rich apex), ruling out that they formed solely via liquid immiscibility

1990; Lee and Wyllie 1997, 1998; Brooker 1998; Brooker and Kjarsgaard 2010). From the phase relations shown in Fig. 13, it becomes obvious that the two carbonated silicate and the phonolitic melts do not represent two melts that exsolved from each other, as two liquids that lie in the field of liquid immiscibility would exsolve along the cotectic lines, so that one composition would lie at the lowermost $\left(\mathrm{SiO}_{2}\right.$ and $\mathrm{Al}_{2} \mathrm{O}_{3}$ rich) two field boundary, and the co-existing composition would lie at the opposite $\left(\mathrm{Na}_{2} \mathrm{O}\right.$ and $\mathrm{K}_{2} \mathrm{O} / \mathrm{CaO}$ rich) two field boundary. Yet, the glasses show striking evidence for liquid immiscibility, such as the vesicular shape of the innermost glass (melt 1). Consequently, if these glasses do represent quenched products of two melts that formed via liquid immiscibility, as indicated by the vesicular shape, then there must have been an additional, $\mathrm{Na}_{2} \mathrm{O}$ - and $\mathrm{K}_{2} \mathrm{O}$-rich liquid that simultaneously separated from the co-existing $\mathrm{SiO}_{2}$ - and $\mathrm{Al}_{2} \mathrm{O}_{3}$-rich liquids, but is now not present in the sapphire. Evidence for the existence of such an $\mathrm{Na}_{2} \mathrm{O}$ - and $\mathrm{K}_{2} \mathrm{O}$-rich liquid is given by the alkali-rich daughter phases in the fluid inclusions that were observed in some of the sapphires. This $\mathrm{Na}_{2} \mathrm{O}$ - and $\mathrm{K}_{2} \mathrm{O}$-rich liquid would have a composition that lies somewhere on the ' $a-b$ ' two liquid field boundary in Fig. 13. Upon cooling, the melt will evolve from a to $b$ via crystallization of silicate phases, and will then evolve along the silicate-carbonate field boundary ' $b-c$ ' with the co-precipitation of silicate and carbonate phases (Lee and Wyllie 1998). Such a melt would be a carbonatite containing $\leq 5 \mathrm{wt} \% \mathrm{SiO}_{2}+\mathrm{Al}_{2} \mathrm{O}_{3}$. The prerequisite for a liquid that lies along the $\mathrm{a}-\mathrm{b}$ two liquid field boundary to cross the silicate liquidus surface and reach the silicate-carbonate field boundary to precipitate carbonate minerals is the physical separation of the carbonatitic melt from the co-existing silicate melt (e.g., Kjarsgaard and Peterson 1991; Lee and Wyllie 1998). When the necessity for the presence of carbonatitic melt is combined with the abundance of carbonate mineral inclusions and $\mathrm{CO}_{2}$-rich fluid inclusions in the sapphires, it seems most probable that the sapphires crystallized from the carbonatite that separated from the melts now present as glass inclusions within the sapphires. After physical separation of the putative carbonatitic melt from the co-existing silicate melt, the carbonatitic melt will evolve separately. It is likely that the sapphires from the SVF crystallized from these carbonatitic melts that exsolved from a highly evolved melt of phonolitic composition.

Additional support to the idea that sapphires crystallized from a carbonatitic parental melt is given by the extremely low viscosity of carbonatitic melts of $\sim 1.5 \times 10^{-2}-5 \times 10^{-3} \mathrm{~Pa}$ s (Dobson et al. 1996) which would promote rapid ion transport through the melt that is needed to form megacrystic corundum at low degrees of alumina oversaturation.

A possible origin of the sapphires from carbonatitic melts could be identified employing oxygen isotope measurements on the sapphires, as carbonate-associated corundum has generally higher $\delta^{18} \mathrm{O}$ values than others (Giuliani et al. 2005). Yet, interpreting oxygen isotope data from sapphires that prospectively precipitated from a carbonatitic melt would be quite difficult as there are no data available about oxygen isotope fractionation between corundum and carbonatitic melt.

\section{Genetic relation between the sapphires and their host rocks}

In situ LA-ICP-MS U-Pb dating of a syngenetic columbite-Mn inclusion within a xenocrystic sapphire hosted by alkaline basalt from the Oelberg in the SVF yielded an age of $24.73 \pm 0.35 \mathrm{Ma}$. This age closely matches the age of alkaline mafic volcanism in the area. The alkali basalts from the Oelberg have an ${ }^{40} \mathrm{Ar}-{ }^{39} \mathrm{Ar}$ isochron age of $24.91 \pm 0.45 \mathrm{Ma}$ (Przybyla 2013) and can thus be regarded as cogenetic with the Siebengebirge sapphires. The Oligocene age of the sapphires confirms a magmatic origin, as a sapphire that originated from the basement below the SVF would be expected to yield a Variscan age. The agreement between the age of the Siebengebirge sapphires and the alkaline basaltic volcanism provides crucial evidence for a genetic link between the processes 
triggering alkaline basaltic magmatism and the crystallization of sapphire megacrysts. The genetic link between the sapphire crystallization and host alkaline basalt must be via $\mathrm{CO}_{2}$. As mentioned in the introduction, the BGY sapphires are restricted in occurrence to a very specific geotectonic setting, i.e., to intra-continental rift-related alkaline basalts. It has been inferred by many previous studies (e.g., Hirose 1997; Green and Falloon 1998; Dasgupta et al. 2007; Zeng et al. 2010) that the mantle source generating continental intra-plate alkaline volcanics must be either carbonate-bearing, or metasomatically enriched by $\mathrm{CO}_{2}$ bearing fluids. Also, the alkaline mafic melts from the SVF are suggested to be generated from refertilized carbonated spinel peridotite (Jung et al. 2012). This infers that BGY sapphires are only generated in intra-continental settings where the mantle source is enriched in $\mathrm{CO}_{2}$, so that primary melts can evolve to phonolites and later expell carbonatites which in turn may crystallize sapphires. The necessity of a $\mathrm{CO}_{2}$ enriched mantle source to produce melts that are capable of precipitating BGY sapphires explains the lack of sapphires in $\mathrm{SiO}_{2}$ saturated basalts such as tholeiites.

\section{Barometry}

When a magmatic mineral contains $\mathrm{CO}_{2}$-bearing fluid inclusions, the density of the inclusions may be used to quantify the pressure during crystallization (Bertrán 1983). The density of $\mathrm{CO}_{2}$-bearing fluid inclusions can be calculated from the distance between the $\mathrm{CO}_{2}$ main peaks at $\sim 1388.0 \mathrm{~cm}^{-1}$ (upper band) and $\sim 1285.5 \mathrm{~cm}^{-1}$ (lower band) of its vibrational spectrum obtained using Raman spectroscopy (e.g., Garrabos et al. 1980; Rosso and Bodnar 1995; Yamamoto and Kagi 2006; Wang et al. 2011). For the Siebengebirge sapphires, we used the equation of Wang et al. (2011) that relates the distance between the $\mathrm{CO}_{2}$ upper and lower band to the density of a $\mathrm{CO}_{2}$-bearing fluid inclusion, and obtained densities in the range of $0.3-0.9 \mathrm{~g} / \mathrm{cm}^{3}$. A systematic relation between the estimated density of a fluid inclusion and its location within the host sapphire was not observed. The pressure during entrapment of the fluid by the host crystal can be calculated from the volumetric properties of the fluid inclusion, given that composition and trapping temperature are known and given that the system was closed. Pressure and temperature vary along isochores. The $\mathrm{P}-\mathrm{V}-\mathrm{T}$ relations of pure $\mathrm{CO}_{2}$ were calculated by Yamamoto et al. (2002) from the equation of state for $\mathrm{CO}_{2}$ by Pitzer and Sterner (1994). Assuming that the Siebengebirge sapphires crystallized from carbonatitic melts and taking average liquidus temperatures for carbonatites

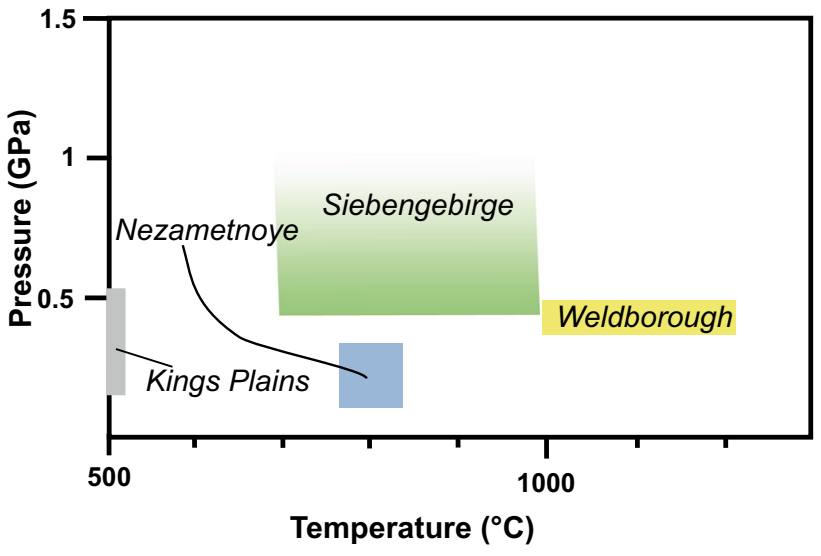

Fig. 14 P-T stability field estimated for the Siebengebirge sapphires. The field for the Siebengebirge sapphires is drawn based on assumed average temperatures of $700-1000{ }^{\circ} \mathrm{C}$ for the carbonatites that crystallized the sapphires (Dobson et al. 1996; Genge et al. 1995; Wolff 1994). For comparison, the P-T fields for the Weldborough (McGee 2005) and Kings Plains (Abduriyim et al. 2014) sapphires in Australia, and sapphires from the Nezametnoye deposit in the Primorsky Region, Far East Russia (Pakhomova et al. 2006) are shown

of $700-1000{ }^{\circ} \mathrm{C}$ (Dobson et al. 1996; Genge et al. 1995; Wolff 1994), the pressure of sapphire crystallization must have been between 0.1 and $0.4 \mathrm{GPa}$. As we cannot exclude the possibility that during ascent-related decompression of the sapphires some fluid inclusions decrepitated to form inclusions of lower density than the original one, we therefore assume that the highest estimated pressure of $0.4 \mathrm{GPa}$ to be the minimum pressure of fluid trapping and hence of sapphire crystallization. This pressure corresponds to a depth of $\sim 12 \mathrm{~km}$.

Based on fluid inclusions, the lithostatic pressure during crystallization has been previously obtained for sapphires from Weldborough and Kings Plains, Australia (McGee 2005; Abduriyim et al. 2014), and from the Nezametnoye deposit in the Primorsky Region, Far East Russia (Pakhomova et al., 2006), and are estimated to be $0.45 \mathrm{GPa}$ (Weldborough), 0.07-0.54 GPa (Kings Plains), and $0.17-0.3 \mathrm{GPa}$ (Nezametnoye), corresponding to midto lower-crustal levels.

The pressures estimated for the Siebengebirge sapphires agree well with pressures estimated for sapphires from Weldborough and Kings Plains, Australia (McGee 2005; Abduriyim et al. 2014) and the Nezametnoye deposit in the Primorsky Region (Pakhomova et al. 2006) (Fig. 14). The temperature estimates for the Nezametnoye $\left(780-820{ }^{\circ} \mathrm{C}\right)$ sapphires are also well in accord with a putative crystallization from carbonatites at low magmatic temperatures. All P-T estimates from the literature, and from this study are well in accord with a crystallization of the magmatic sapphires from carbonatitic melts at mid-crustal levels. 


\section{Petrogenetic model for the Siebengebirge sapphires}

The formation of the alkaline basalt-hosted sapphires from the SVF is a result of a complex line of magmatic processes that elapsed during a very short time scale. During Miocene times, extensive active volcanism started in the SVF, producing a broad spectrum of $\mathrm{SiO}_{2}$ saturated and undersaturated primitive and evolved volcanic rocks. The alkaline mafic melts probably formed due to melting of amphibole and phlogopite-bearing peridotite in the spinel peridotite stability zone (Jung et al. 2012; Schubert et al. 2015), at pressures between 1 and $2.5 \mathrm{GPa}$ and temperatures between 1150 and $1350{ }^{\circ} \mathrm{C}$ (Fig. 15a). These $\mathrm{P}-\mathrm{T}$ conditions correspond to the lithospheric mantle. It has been repeatedly suggested that the alkaline mafic rocks were formed from a carbonatite-metasomatized mantle source (Jung et al. 2012; Pfänder et al. 2012). As a result, the melts must have been enriched in volatiles including $\mathrm{CO}_{2}$. With ensuing fractionation of the primitive melts towards more evolved phonolitic compositions, the partial pressure of $\mathrm{CO}_{2}\left(\mathrm{pCO}_{2}\right)$ in the melt increases (Fig. 15b) until at high degrees of differentiation and high $\mathrm{pCO}_{2}$, a carbonatitic melt exsolves from the phonolite (Kogarko 1997). The carbonatitic melt exsolving from a highly evolved phonolite will be $\mathrm{CaO}$ - and volatile-rich but is expected to be depleted in $\mathrm{SiO}_{2}, \mathrm{MgO}$, and $\mathrm{FeO}$ (Kogarko 1997). The carbonatite will cool and start to crystallize carbonate phases when it is physically separated from the co-existing silicate melt (Lee and Wyllie 1998). The physical separation of the carbonatitic melt from the silicate melt will probably occur rapidly after exsolution due to the significant viscosity and density difference of both melts. The crystallization of the sapphires from highly fractionated carbonatite occurred at middle- to upper crustal levels (Fig. 15c) after the physical separation of the carbonatite and the phonolite melt, as indicated by the presence of carbonate inclusions in the sapphires. A fresh pulse of hot ascending alkali basalts later encounters the carbonatite melt pocket in the crust on their way upwards, decomposes the carbonatitic melt and incorporates the sapphires to transport them to the surface (Fig. 15d).

\section{Summary and conclusions}

Magmatic sapphires are well known to occur associated with intra-continental alkaline mafic volcanism. Around the world, predominantly in Asia and Australia, many alkaline basalt fields are reported to carry megacrysts of magmatic sapphires. To date, many hypotheses have been proposed to investigate the nature of these so-called BGY sapphires. Still, although these studies comprise detailed investigations on the sapphires including trace element analyses, analysis of mineral inclusions as well as melt and/or fluid inclusion and oxygen isotope measurements, no consensus was reached about the origin of these sapphires. Moreover, these studies cannot explain the intimate association of blue-green-yellow (BGY) sapphires with alkaline basaltic volcanism. This study on the BGY sapphires hosted by alkaline basalts from the Siebengebirge Volcanic Field (SVF) elucidates many yet unknown details about the formation of this special type of gemstone. The main conclusions from this study can be summarized as follows:

1) Sapphire megacrysts are xenogenetic to the host basalts, as indicated by the spinel corona surrounding each sapphire at the contact towards the basalt.

2) The parental melt was probably a carbonatitic melt that exsolved from a highly evolved phonolitic melt, as evidenced by carbonate inclusions, strong enrichment in the HFSE, by the glass inclusions of carbonated silicate in contact with phonolite, and by $\mathrm{CO}_{2}$-bearing fluid inclusions.

3) There is a close genetic relation between the sapphires and their host alkaline mafic rocks, as evidenced by the overlapping age of the Siebengebirge sapphires dated in this work and the age of the alkaline mafic volcanism in the area (Przybyla 2013). We identified the genetic link between magmatic sapphires and the host basalt that has been a puzzle to date, to bear upon $\mathrm{CO}_{2}$. Only alkaline volcanic suites can build up enough $\mathrm{CO}_{2}$ in the magma chamber upon fractionation so that at high degrees of fractionation a carbonatitic melt exsolves which in turn can crystallize sapphires at a late stage of evolution. During ascent, fresh alkaline basaltic melts that encounter carbonatite melt pockets will decompose the carbonatite and incorporate the sapphires to carry them to the surface.

Our findings from the Siebengebirge sapphires raise the question about an overall applicability of the genetic model to worldwide occurrences of magmatic sapphires. It is hard to tell if all magmatic sapphires from the world must have precipitated from a carbonatitic melt that exsolved from highly fractionated $\mathrm{SiO}_{2}$ undersaturated silicate melts. If so, one would expect more sapphires from other locations to show similar features evidencing for a carbonatitic parental melt. Yet, it is important to notice that even those sapphires from this study do not all show the whole range of features pointing towards a carbonatitic parentage, i.e., carbonate and HFSE-rich mineral inclusions $+\mathrm{CO}_{2}$ fluid inclusions with carbonate daughter phases + silicate/carbonate melt 
Fig. 15 Petrogenetic model for the Siebengebirge sapphires. a Alkaline basaltic volcanism is initiated in the SVF. b Differentiation of alkaline mafic magmas leads to the formation of highly evolved phonolites, which is accompanied by a build-up of $\mathrm{pCO}_{2}$ in the melt. c In the highly evolved phonolite that is now saturated in $\mathrm{CO}_{2}$, a carbonatitic melt exsolves and separates from the phonolite. Sapphires precipitate from the carbonatite after physical separation. $\mathbf{d}$ Fresh pulses of alkaline mafic melts ascend. When encountering the carbonatite lenses, the alkaline mafic melts decompose the carbonatite, incorporate the sapphires and carry them to the surface
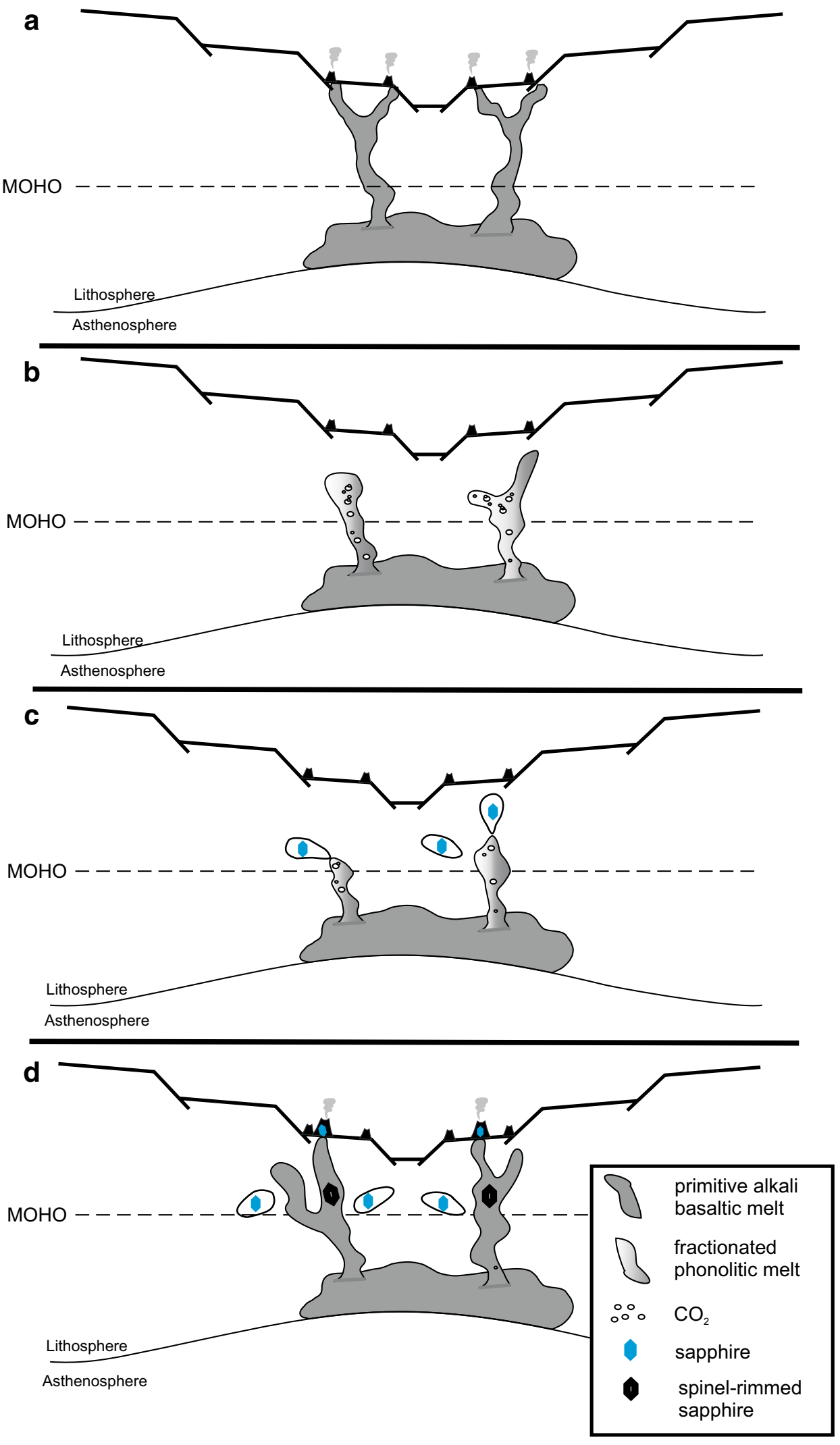
inclusions. It is rather the sum of single pieces of mineralogical evidence from the whole sample set that inferred a carbonatite source for the Siebengebirge sapphires. Therefore, it cannot be excluded that other magmatic sapphires that do not bear mineralogical evidence for a carbonatite origin do not have the same petrogenetic history. Hence, an overall applicability of our petrogenetic model for the origin of magmatic sapphires should be considered, but cannot be stated with certainty. However, from the findings of not only our study, but also from referenced herein, we can state with some confidence that in general, sapphire crystallizing melts were produced via melting of $\mathrm{CO}_{2}$-rich, probably carbonatite metasomatized, source rocks.

Acknowledgements Open access funding provided by University of Vienna. We would like to thank Renate Schumacher for providing the sapphire samples from the mineralogical collection of the University of Bonn, and Nils Jung for sample preparation. Furthermore, we would like to thank two anonymous reviewers for their constructive comments, helping to improve the manuscript. This is contribution no 44 of the DFG funded LA-ICP-MS laboratory of the Steinmann Institute, University of Bonn.

Open Access This article is distributed under the terms of the Creative Commons Attribution 4.0 International License (http://creativecommons.org/licenses/by/4.0/), which permits unrestricted use, distribution, and reproduction in any medium, provided you give appropriate credit to the original author(s) and the source, provide a link to the Creative Commons license, and indicate if changes were made.

\section{References}

Abduriyim A, Sutherland FL, Belousova EA (2012) U-Pb age and origin of gem zircon from the New England sapphire fields, New South Wales, Australia. Aust J Earth Sci 59:1067-1081

Abduriyim A, Kamegata N, Nonguchi N, Kagi H, Sutherland FL, Coldham T (2014) Residual pressure distribution and visualization of mineral inclusions in corundum: application of photoluminescence spectroscopy in relation to sapphires from New England, New South Wales, Australia. Aust Gemmol 25:245-254

Aspen P, Upton BGJ, Dicken AP (1990) Anorthoclase, sanidine and associated megacrysts in Scottish alkali basalts: high pressure syenitic debris from upper mantle sources? Eur J Mineral 2:503-517

Berg RB (2007) Sapphires in the Butte-Deer Lodge Area, Montana. Montana Bur Mines Geol Bull 134, p 59

Berg RB (2014) Sapphires in the southwestern part of the Rock Creek sapphire district. Montana, Bur Mines Geol Bull, p 135

Berger AL, Berg RB (2006) The Silver Bow sapphire occurrence, Montana: evidence for a volcanic bedrock source for Montana's alluvial sapphire deposits. Econ Geol 101:679-684

Bertrán JF (1983) Study of the Fermi doublet $v_{1}-2 v_{2}$ in the Raman spectra of $\mathrm{CO}_{2}$ in different phases. Spectrochim Acta 39A:119-121

Brauns R (1922) Die Mineralien der Niederrheinischen Vulkangebiete mit besonderer Berücksichtigung ihrer Bildung und Umbildung. Schweizerbart, Stuttgart

Brooker RA (1998) The effect of $\mathrm{CO}_{2}$ saturation on immiscibility between silicate and carbonate liquids: an experimental study. J Petrol 39:1905-1915
Brooker RA, Hamilton DL (1990) Three-liquid immiscibility and the origin of carbonatites. Nature 346:459-462

Brooker RA, Kjarsgaard BA (2010) Silicate-carbonate liquid immiscibility and phase relations in the system $\mathrm{SiO}_{2}-\mathrm{Na}_{2} \mathrm{O}-\mathrm{Al}_{2} \mathrm{O}_{3}-\mathrm{CaO}-\mathrm{CO}_{2}$ at $0.1-2.5 \mathrm{GPa}$ with applications to carbonatite genesis. J Petrol 52:1281-1305

Che X-D, Wu F-Y, Wang R-Ch, Gerdes A, Ji W-Q, Zhao Z-H, Yang J-H, Zhu Z-Y (2015) In situ U-Pb isotopic dating of columbitetantalite by LA-ICP-MS. Ore Geol Rev 65:979-989

Coenraads RR (1990) Key areas for alluvial diamond and sapphire exploration in the New England gem fields, New South Wales, Australia. Econ Geol 85:1186-1207

Coenraads RR, Sutherland FL, Kinny PD (1990) The origin of sapphires: U-Pb dating of zircon inclusions shed new light. Miner Mag 54:113-122

Dasgupta R, Hirschmann MM, Smith ND (2007) Partial melting experiments of peridotite $+\mathrm{CO}_{2}$ at $3 \mathrm{GPa}$ and genesis of alkalic ocean island basalts. J Petrol 48:2093-2124

Dill HG, Gerdes A, Weber B (2007) Cu-Fe-U phosphate mineralization of the Hagendorf-Pleystein pegmatite province, Germany: with special reference to laser-ablation-inductive-coupledplasma mass spectrometry (LA-ICP-MS) of limonite-cored torberite. Miner Mag 71:423-439

Dobson DP, Jones AP, Rabe R, Toshimori S, Kurita K, Taniguchi T, Kondo T, Kato T, Shimomura O, Urakawa S (1996) In-situ measurement of viscosity and density of carbonate melts at high pressure. Earth Planet Sci Lett 143:207-215

Freestone IC, Hamilton DL (1980) The role of liquid immiscibility in the genesis of carbonatites-an experimental study. Contrib Miner Petr 73:105-117

Gäbler H-E, Melcher F, Graupner T, Bahr A, Sitnikova M, HenjesKunst F, Oberthür T, Brätz H, Gerdes A (2011) Speeding up the analytical workflow for coltan finger-printing by an integrated mineral liberation analysis/LA-ICP-MS approach. Geostand Geoanal Res 35:431-448

Garland MI (2002) The alluvial Sapphire Deposits of Western Montana. (Ph.D. Thesis). University of Toronto

Garnier V, Ohnenstetter D, Giuliani G, Fallick AE, Phan Trong T, Hoang Quang V, Van Pham L, Schwarz D (2005) Basalt petrology, zircon ages and sapphire genesis from Dak Nong, southern Vietnam. Miner Mag 69:21-38

Garrabos Y, Tufeu R, Le Neindre B, Zalczer G, Beysens D (1980) Rayleigh and raman scattering near the critical point of carbon dioxide. J Chem Phys 72:4637-4651

Genge MJ, Price GD, Jones AP (1995) Molecular dynamics simulations of $\mathrm{CaCO}_{3}$ melts to mantle pressures and temperatures: implications for carbonatite magmas. Earth Planet Sci Lett 131:225-238

Gerdes A, Zeh A (2006) Combined U-Pb and Hf isotope LA(MC-)-ICP-MS analyses of detrital zircons: comparison with SHRIMP and new constraints for the provenance and age of an Armorican metasediment in Central Germany. Earth Planet Sci Lett 249:47-61

Gerdes A, Zeh A (2009) Zircon formation versus zircon alteration-new insights from combined $\mathrm{U}-\mathrm{Pb}$ and $\mathrm{Lu}-\mathrm{Hf}$ in situ LA-ICP-MS analyses, and consequences for the interpretation of Archean zircon from the Central Zone of the Limpopo Belt. Chem Geol 261:230-243

Giuliani G, Fallick AE, Garnier V, France-Lanord Ch, Ohnestetter D, Schwarz D (2005) Oxygen isotope composition as a tracer for the origin of ruby and sapphires. Geology 33:249-252

Giuliani G, Fallick AE, Rakotondrazafy M, Ohnestetter D, Andriamamonjy A, Rakotosamizanay S, Ralantoarison Th, Razanatseheno M, Dungaigre Ch, Schwarz D (2007) Oxygen isotope systematic of gem corundum deposits in 
Madagascar: relevance for their geological origin. Miner Depos 42:251-270

Giuliani G, Fallick A, Ohnestetter D, Pegere G (2009) Oxygen isotopes composition of sapphires from the French Massif Central: implications for the origin of gem corundum in basaltic fields. Miner Depos 44:221-231

Giuliani G, Ohnestetter D, Fallick AE, Groat LA, Fagan J (2014) The geology and genesis of gem corundum. In: Groat LA (ed) Geology of gem deposits, 2nd edn. Mineralogica Association of Canada, Short Course Series 44, pp 29-112

Giuliani G, Pivin M, Fallick AE, Ohnestetter D, Song Y, Demaiffe D (2015) Geochemical and oxygen isotope signatures of mantle corundum megacrysts from the Mbuji-Mayi kimberlite, Democratic Republic of Congo, and the Changle alkali basalt, China. CR Geosci 347:24-34

Graham IT, Sutherland FL, Web GB, Fanning CM (2004) Polygenetic corundums from New South Wales gemfields. In: Khanchuk AI, Gonevchuk GA, Mitrokhin AN, Simaneko IF, Cook NJ, Seltmann R (eds) Metallogeny of the Pacific Northwest: Tectonics, magmatism and metallogeny of active continental margins. Dalnauka, Vladivostok, pp 336-339

Graham IT, Sutherland L, Zaw K, Nechav V, Khanchuk A (2008) Advances in our understanding of the gem corundum deposits of the West Pacific continental margins intraplate basaltic fields. Ore Geol Rev 34:200-215

Green DH, Falloon TJ (1998) Pyrolite: a Ringwood concept and its current expression. In: Jackson I (ed) The Earth's mantle: composition, structure, and evolution. Cambridge University Press, Cambridge, pp 311-378

Guo J, Wang F, Yakoumelos G (1992) Sapphires from Changle in Shandong Province, China. Gems Gemol 28:255-260

Guo J, O'Reilly SY, Griffin WL (1996) Corundum from basaltic terrains: a mineral inclusion approach to the enigma. Contrib Miner Petr 122:368-386

Hamilton DL, Freestone IC (1979) Origin of carbonatites by liquid immiscibility. Nature 279:52-54

Hirose K (1997) Partial melt compositions of carbonated peridotite at $3 \mathrm{GPa}$ and role of $\mathrm{CO}_{2}$ in alkali-basalt magma generation. Geophys Res Lett 24:2837-2840

Hughes RW (1997) Ruby and sapphire. RWH Publishing, Boulder Co, USA

Illies JH, Prodehl C, Schmincke H-U, Semmel A (1979) The quarternary uplift of the rhenish shield in Germany. Tectonophysics 61:197-225

Irving AJ (1986) Polybaric magma mixing in alkali basalts and kimberlites: evidence from corundum, zircon and ilmenite megacrysts. In: 4th International Kimberlite Conference, Perth, Geological Society of Australia Abstr. Ser., vol. 16. Blackwell Scientific, Oxford, pp 262-264

Ishitani T, Ohnishi T, Kawanami Y (1990) Micromachining and device transplantation using focused ion beam. Jpn J Appl Phys 29(10R):2283

Izokh AE, Smirnov SZ, Egorova VV, Anh TT, Kovyazin SV, Phuong NT, Kalinina VV (2010) The conditions of formation of sapphire and zircon in the areas of alkali-basaltoid volcanism in Central Vietnam. Russ Geol Geophys 51:719-733

Jackson SE, Pearson NJ, Griffin WL, Belousova EA (2004) The application of laser ablation-inductively coupled plasma-mass spectrometry to in situ U-Pb zircon geochronology. Chem Geol 211:47-69

Jochum KP, Weis U, Stoll B et al (2011) Determination of reference values for NIST SRM 610-617 glasses following ISO guidelines. Geostan Geoanal Res 35:397-429

Jung S, Vieten K, Romer RL, Mezger K, Hoernes S, Satir M (2012) Petrogenesis of tertiary alkaline magmas in the Siebengebirge, Germany. J Petrol 53:2381-2409
Junge M, Oberthür T, Melcher F (2014) Cryptic variation of chromite chemistry, platinum group element and platinum group mineral distribution in the UG-2 chromitite: an example from the Karee Mine, western Bushveld Complex, South Africa. Econ Geol 109:795-810

Khamloet P, Pisutha-Arnond V, Sutthirat C (2014) Mineral inclusions in sapphire from the basalt-related deposit in Bo Phloi, Kanchanaburi, western Thailand: indication of their genesis. Russ Geol Geophys 55:1087-1102

Kiefert L, Schmetzer K (1987) Blue and yellow sapphire from Kaduna Province, Nigeria. J Gemmol 22:484-496

Kjarsgaard B, Hamilton DL (1988) Liquid immiscibility and the origin of alkali-poor carbonatites. Miner Mag 52:43-55

Kjarsgaard B, Peterson T (1991) Nephelinite-carbonatite liquid immiscibility at Shombole volcanoe, East Africa: petrographic and experimental evidence. Miner Petrol 43:293-314

Kogarko LN (1997) Role of $\mathrm{CO}_{2}$ on differentiation of ultramafic alkaline series: liquid immiscibility in carbonate-bearing phonolitic dykes (Polar Siberia). Miner Mag 61:549-556

Kolb M, Paulik H, Kirchenbaur M, Münker C (2012) Petrogenesis of mafic to felsic lavas from the Oligocene Siebengebirge Volcanic Field (Germany): implications for the origin of intracontinental volcanism in Central Europe. J Petrol 53:2349-2379

Koster van Groos AK, Wyllie PJ (1963) Experimental data bearing on the role of liquid immiscibility in the genesis of carbonatites. Nature 199:801-802

Koster van Groos AK, Wyllie PJ (1966) Liquid immiscibility in the system $\mathrm{Na}_{2} \mathrm{O}-\mathrm{Al}_{2} \mathrm{O}_{3}-\mathrm{SiO}_{2}-\mathrm{CO}_{2}$ at pressures to 1 kilobar. Am J Sci 264:234-255

Krzemnicki M, Hänni HA, Guggenheim R, Mathys D (1996) Investigations on sapphires from an alkali basalt, South West Rwanda. J Gemmol 25:90-106

Le Maitre RW (2002) A classification and glossary of terms; recommendations of the International Union of Geological Sciences: Subcommision on the systematics of igneous rocks. Cambridge University Press, Cambridge

Lee W-J, Wyllie PJ (1997) Liquid immiscibility between nephelinite and carbonatite from 1.0 to $2.5 \mathrm{GPa}$ compared with mantle melt compositions. Contrib Miner Petr 127:1-16

Lee W-J, Wyllie PJ (1998) Process of crustal carbonatite formation by liquid immiscibility and differentiation, elucidated by model systems. J Petrol 39:2005-2013

L'Heureux I (2013) Self-organized rhythmic patterns in geochemical systems. Philos Trans R Soc Lon Math Phys Eng Sci 371(2004):20120356

Limtrakun P, Zaw K, Ryan CG, Mernagh TP (2001) Formation of the Denchai gem sapphires, northern Thailand: evidence from mineral chemistry and fluid/melt inclusion characteristics. Miner Mag 65:725-735

Linthout K, Paulick H, Wijbrans JR (2009) Provenance of basalt blocks from Roman sites in Vleuten-De Meern (The Netherlands) traced to the Tertiary Siebengebirge (Germany): a geoarcheological quest using petrological and geochemical methods. Geol Mijnbouw 88:78-99

Liu T-C, Presnall DC (1990) Liquidus phase relationships on the join anorthite-forsterite-quartz at $20 \mathrm{kbar}$ with applications to basalt petrogenesis and igneous sapphirine. Contrib Miner Petrol 104:735-742

Liu T-C, Presnall DC (2000) Liquidus phase relations in the system $\mathrm{CaO}-\mathrm{MgO}-\mathrm{Al}_{2} \mathrm{O}_{3}-\mathrm{SiO}_{2}$ at $2.0 \mathrm{GPa}$ : applications to basalt fractionation, eclogites, and igneous sapphirine. J Petrol 41:3-20

MacNevin AA (1972) Sapphires in the New England district, New South Wales. Records of the geological survey of New South Wales 14:19-35 
Malikova P (1999) Origin of the alluvial sapphires from the Jizerska Louka alluvial deposits in North Bohemia, Czech Republic, Europe. Aust Gemol 20:202-206

McGee BM (2005) Characteristics and origin of the Weldborough sapphire, NE Tasmania. (Unpublished BSc thesis): University of Tasmania, School of Earth Science, Hobart, Australia

Melcher F, Sitnikova MA, Graupner T, Martin N, Oberthür T (2008) Analytical fingerprint of columbite-tantalite (coltan) mineralization in pegmatites: focus on Africa. In: Proceedings, Ninth International Congress for Applied Mineralogy (ICAM), pp $615-624$

Melcher F, Graupner T, Gäbler H-E, Sitnikova M, Henjes-Kunst F, Oberthür T, Gerdes A, Dewaele S (2015) Tantalum-(niobiumtin) mineralisation in African pegmatites and rare metal granites: constraints from $\mathrm{Ta}-\mathrm{Nb}$ oxide mineralogy, geochemistry and U-Pb geochronology. Ore Geol Rev 64:667-719

Merino E, Wang Y (2001) Geochemical self-organization in rocks: occurrences, observations, modeling, testing-with emphasis on agate genesis. Yearb Self-Org 11:13-45

Milholland CS, Presnall DC (1998) Liquidus phase relations in the $\mathrm{CaO}-\mathrm{MgO}-\mathrm{Al}_{2} \mathrm{O}_{3}-\mathrm{SiO}_{2}$ system at $3.0 \mathrm{GPa}$ : the aluminous pyroxene thermal divide and high-pressure fractionation of picritic and komatiitic magmas. J Petrol 39:3-27

Nechaev VP, Nechaeva EV, Chashchin AA, Vysotskiy SV, Graham IT, Sutherland FL (2009) New isotopic data on late Cenozoic age and mantle origin of gem corundum from placers of Primorye, Russia. Geology 429A:1426-1429

Ohnishi T, Kawanami Y, Ishitani T (1990) Proposal for device transplantation using a focused ion beam. Japan J Appl Phys 29(1A):L188

Oncken O, Winterfeld C, Dittmar U (1999) Accretion of a rifted passive margin: the Late Paleozoic Rhenohercynian fold and thrust belt (Middle European Variscides). Tectonics 18:75-91

Ortoleva PJ (1994) Geochemical self-organization (p 411). Oxford University Press, Clarendon Press, Oxford

Pakhomova VA, Zalishchak BL, Odarichenko EG, Lapina MI, Karmanov NS (2006) Study of melt inclusions in the Nezametnoye corundum deposit, Primorsky region of the Russian Far East: petrogenetic consequences. J Geochem Explor 89:302-305

Palke AC, Renfro NB, Berg RB (2015) Glassy melt inclusions in sapphires from the Rock Creek and Missouri River deposits in Montana, USA. Geol Soc Am Abstr Progr 47:200

Palke AC, Renfro ND, Berg RB (2016) Origin of sapphires from a lamprophyre dike at Yogo Gulch, Montana, USA: clues from their melt inclusions. Lithos 260:339-344

Palke AC, Renfro ND, Berg RB (2017) Melt inclusions in alluvial sapphires from Montana, USA: formation of sapphires as a restitic component of lower crustal melting? Lithos 278-281:43-53

Pardieux V, Sansawong S, Mayal J, Sturman N (2014) Blue sapphires from the Mambilla Plateau, Taraba State, Nigeria. A preliminary exploration. GIA News from Research, GIA Laboratory Bangkok

Peucat JJ, Ruffault P, Fritsch E, Bouhnik-Le Coz M, Simonet C, Lasnier B (2007) $\mathrm{Ga} / \mathrm{Mg}$ ratio as a new geochemical tool to differentiate magmatic from metamorphic blue sapphires. Lithos 98:261-274

Pfänder JA, Jung S, Münker C, Stracke A, Mezger K (2012) A possible high $\mathrm{Nb}$ /Ta reservoir in the continental lithospheric mantle and consequences on the global $\mathrm{Nb}$ budget-Evidence from continental basalts from Central Germany. Geochim Cosmochim Ac 77:232-251

Pitzer KS, Sterner SM (1994) Equations of state valid continuously from zero to extreme pressures for $\mathrm{H}_{2} \mathrm{O}$ and $\mathrm{CO}_{2}$. J Chem Phys 101:3111-3116

Przybyla M (2013) ${ }^{40} \mathrm{Ar} /{ }^{39} \mathrm{Ar}-$ Datierungen an Vulkaniten des Siebengebirges. Msc thesis, University of Cologne, Germany
Rosso KM, Bodnar RJ (1995) Microthermometric and Raman spectroscopic detection limits of $\mathrm{CO}_{2}$ in fluid inclusions and the Raman spectroscopic characterization of $\mathrm{CO}_{2}$. Geochim Cosmochim Ac 59:3961-3975

Saminpanya S (2001) Ti-Fe mineral inclusions in star sapphires from Thailand. Aust Gemmol 21:125-128

Schubert S, Jung S, Pfänder JA, Hauff F, Garbe-Schönberg D (2015) Petrogenesis of Tertiary continental intra-plate lavas between Siebengebirge and Westerwald, Germany: constraints from trace element systematics and $\mathrm{Nd}, \mathrm{Sr}$ and $\mathrm{Pb}$ isotopes. J Volcanol Geoth Res 305:84-99

Schwarz D, Kanis J, Schmetzer K (2000) Sapphires from Antsiranana Province, Northern Madagascar. Gems Gemol 36:216-233

Sen G, Presnall DC (1984) Liquidus phase relations on the join anorthite-forsterite-quartz at $10 \mathrm{kbar}$ with applications to basalt petrogenesis. Contrib Mineral Petrol 85:404-408

Shore R, Weldon JL (2009) Ruby and sapphire production and distribution: a quarter century of change. Gems Gemol 45:236-259

Simonet C, Fritsch E, Lasnier B (2008) A classification of gem corundum deposits aimed towards gem exploration. Ore Geol Rev 34:127-133

Smith SR, Foster GL, Romer RL, Tindle AG, Kelley SP, Noble SR, Horstwood M, Breaks FW (2004) U-Pb columbite-tantalite chronology of rare-element pegmatites using TIMS and Laser Ablation-Multi-Collector-ICP-MS. Contrib Miner Petrol 147:549-564

Song Y, Hu W (2009) Carbonates and sulfates-bearing melt inclusions in corundum megacrysts from Changle basalts of Shandong province and their implications. Acta Petrol Miner 28:349-363

Stacey JT, Kramers JD (1975) Approximation of terrestrial lead isotope evolution by a two-stage model. Earth Planet Sci Lett 26(2):207-221

Stephenson PJ (1976) Sapphire and zircon in some basaltic rocks from Queensland, Australia. In: Abstracts 25th International Geological Congress, vol. 2

Sutherland FL (1996) Alkaline rocks and gemstones, Australia: a review and synthesis. Aust J Earth Sci 43:323-343

Sutherland FL, Schwarz D (2001) Origin of gem corundums from basaltic fields. Aust Gemmol 21:30-33

Sutherland FL, Schwarz D, Jobbins EA, Coenraads RR, Webb G (1998a) Distinctive gem corundum suites from discrete basalt fields: a comparative study of Barrington, Australia, and West Pailin, Cambodia, gemfields. J Gemmol 26:65-85

Sutherland FL, Hoskin POW, Fanning CM, Coenraads RR (1998b) Models of corundum origin from alkali basaltic terrains: a reappraisal. Contrib Miner Petrol 133:356-372

Sutherland FL, Graham IT, Pogson RE, Schwarz D, Webb GB, Coenraads RR, Fanning CM, Hollis JD, Allen TC (2002) The Tumbarumba basaltic gem field, New South Wales. In relation to sapphire-ruby deposits of eastern Australia. Rec Aust Mus 54:215-248

Sutherland FL, Duroc-Danner JM, Meffre S (2008) Age and origin of gem corundum and zircon megacrysts from the Mercades-Rio Mayo area, South-west Colombia, South America. Ore Geol Rev 34:155-168

Sutherland FL, Giuliani G, Fallick AE, Garland M, Webb G (2009a) Sapphire and ruby characteristics, West Palin, Cambodia: clues to their origin based on trace element and $\mathrm{O}$ isotope analysis. Aust Gemmol 23:373-432

Sutherland FL, Zaw K, Meffre S, Giuliani G, Fallick AE, Graham IT, Webb GB (2009b) Gem-corundum megacrysts from eastern Australian basalt fields: trace elements, oxygen isotopes and origins. Aust J Earth Sci 6:1003-1022

Sutherland FL, Coenraads RR, Abduriym A, Meffre S, Hoskin PWO, Giuliani G, Beattie R, Wuhrer R, Sutherland GB (2015) 
Corundum (sapphire) and zircon relationships, Lava Plains gem fields, NE Australia: integrated mineralogy, geochemistry, age determination, genesis and geographical typing. Miner Mag 79:545-581

Sutherland FL, Graham IT, Harris SJ, Coldham T, Powell W, Belousova EA, Martin L (2017) Unusual ruby-sapphire transition in alluvial megacrysts, Cenozoic basaltic gem field, New England, New South Wales, Australia. Lithos 278:347-360

Todt W, Lippolt HJ (1980) K-Ar age determination on Tertiary volcanic rocks: V Siebengebirge, Siebengebirge-Graben. J Geophys-Z Geophys 48:18-27

Uher P, Giuliani G, Szakall S, Fallick A, Strunga V, Vaculovic T, Ozdin D, Greganova M (2012) Sapphires related to alkali basalts from the Cerová Highlands, Wesatern Carpathians (southern Slovakia): composition and origin. Geol Carpath 63:71-82

Van Long P, Quang Vinh H, Garnier V, Giuliani G, Ohnestetter D, Lhomme T, Schwarz D, Fallick AE, Dubessy J, Trong Trinh P (2004) Gem corundum deposits in Vietnam. J Gemmol 29:129-147

Vichit P (1987) Gemstones in Thailand. J Geol Soc Thailand 9:108-133

Vieten K, Hamm HM, Grimmeisen W (1988) Tertiärer Vulkanismus des Siebengebirges. Fortschr Der Mineral Beih 66:1-42

Vysotskij SV, Barkar AV (2009) Sapphire of the Primorje Region. Dal'nauka, Vladivostok (in Russian)

Wang X, Chou I-M, Hu W, Burruss RC, Sun Q, Song Y (2011) Raman spectroscopic measurements of $\mathrm{CO}_{2}$ density: experimental calibration with high-pressure optical cell (HPOC) and fused silica capillary capsule (FSCC) with application to fluid inclusion observations. Geochim Cosmochim Ac 75:4080-4093

Wedepohl KH, Gohn E, Hartmann G (1994) Cenozoic alkali basaltic magmas of western Germany and their products of differentiation. Contrib Miner Petrol 115:253-278

Whalen JB, Curie KL, Chappell BW (1987) A-type granites: geochemical characteristics, discrimination and petrogenesis. Contrib Miner Petrol 95:407-419

Whitney D, Evans B (2010) Abbreviations for names of rock-forming minerals. Am Miner 95:185-187

Wilson M, Downes H (1991) Tertiary-Quarternary extension related alkaline magmatism in western and central Europe. J Petrol 32:811-849
Wirth R (2004) Focused Ion Beam (FIB) A novel technology for advanced application of micro- and nanoanalysis in geosciences and applied mineralogy. Eur J Miner 16:863-876

Wirth R (2009) Focused Ion Beam (FIB) combined with SEM and TEM: advanced analytical tools for studies of chemical composition, microstructure and crystal structure in geomaterials on a nanometre scale. Chem Geol 261:217-229

Wirth R, Reid D, Schreiber A (2013) Nanometer-sized platinumgroup minerals (PGM) in base metal sulfides: new evidence for an orthomagmatic origin of the Merensky Reef PGE ore deposit, Bushveld Complex, South Africa. Can Miner $51: 143-155$

Wolff JA (1994) Physical properties of carbonatite magmas inferred from molten salt data, and application to extraction patterns from carbonatite-silicate magma chambers. Geol Mag 131:145-153

Wright JB (1985) Geology and mineral resources of West Africa. Allen and Unwin, London

Yamamoto J, Kagi H (2006) Extended micro-Raman densimeter for $\mathrm{CO}_{2}$ applicable to mantle-originated fluid inclusions. Chem Lett 35:610-611

Yamamoto J, Kagi H, Kaneoka I, Lai Y, Prikhod'ko VS, Arai S (2002) Fossil pressures of fluid inclusions in mantle xenoliths exhibiting rheology of mantle minerals: implications for the geobarometry of mantle minerals using micro-Raman spectroscopy. Earth Planet Sci Lett 198:511-519

Yui TF, Wu CM, Limtrakum P, Sricharn W, Boonsoog A (2006) Oxygen isotope studies on placer sapphire and ruby in the Chantaburi-Trat alkali basaltic gemfield, Thailand. Lithos 86:197-211

Zaw K, Sutherland FL, Dellapasqua F, Ryan CG, Yui T-F, Mernagh TP, Duncan D (2006) Contrasts in gem corundum characteristics, eastern Australian basaltic fields: trace elements, fluid/melt inclusions and oxygen isotopes. Miner Mag 70:669-687

Zelong D, Xiaoming C, Wenxuan H, RuCheng W, Ming Z (2007) Coronas of corundum megacrysts in the Neogene Changle basalt and its forming model. Acta Petrol Sinca 23:805-816

Zeng G, Chen L-H, Xu X-S, Jiang S-Y, Hofmann AW (2010) Carbonated mantle sources for Cenozoic intra-plate alkaline basalts in Shandong, North China. Chem Geol 273:35-45

Ziegler PA (1992) European Cenozoic rift system. Tectonophysics 208:91-111 